\title{
Innovationen in der Klimaschutzpolitik deutscher Großstädte
}

\author{
Der Einfluss überlokalen Handelns im Vergleich
}

\author{
Jörg Kemmerzell • Anne Hofmeister
}

Online publiziert: 3. Dezember 2018

(C) Der/die Autor(en) 2018

Zusammenfassung Lokale Klimapolitik wird von zahlreichen Faktoren beeinflusst. Bisherige Studien haben den Einfluss überlokaler Aktivitäten auf die Diffusion innovativer Klimapolitiken jedoch kaum berücksichtigt. Der vorliegende Beitrag untersucht systematisch den Einfluss struktureller Kontextfaktoren und politischer Unternehmer sowie überlokaler Aktivitäten kommunaler Akteure. Die Analyse erfolgt mittels einer mehrstufigen, qualitativ-komparativen Untersuchungsstrategie (Crisp Set QCA). Die Ergebnisse der Untersuchung zeigen, dass strukturelle Faktoren die Extensivität von Klimaschutzmaßnahmen in Großstädten nur begrenzt erklären können. Die Berücksichtigung überlokalen Handelns und politischer Unternehmer führt zu einer erhöhten Erklärungskraft, da diese Faktoren günstige Rahmenbedingungen verstärken oder in einigen Fällen sogar eine substituierende Wirkung entfalten können.

Schlüsselwörter Lokale Politik · Klimapolitik · Überlokales Handeln · QCA · Diffusion von Innovationen

\section{Innovations in Climate Change Policy of German Major Cities}

The Influence of Trans-local Action in Comparison

\begin{abstract}
Local climate policy is influenced by many factors. However, previous studies have barely considered the impact of trans-local activities on the diffusion of innovative climate policies. The article systematically examines the influence of structural contextual factors and political entrepreneurs as well as trans-local activ-
\end{abstract}

Zusatzmaterial online Zusätzliche Informationen sind in der Online-Version dieses Artikels (https:// doi.org/10.1007/s11615-018-0134-4) enthalten.

J. Kemmerzell $(\bowtie) \cdot$ A. Hofmeister

Darmstadt, Deutschland

E-Mail: kemmerzell@pg.tu-darmstadt.de 
ities of cities. The analysis is conducted by means of a qualitative-comparative research design applying a two-stage crisp set QCA. The results of the study show that structural factors can only partially explain the extensiveness of climate protection measures in major cities. The inclusion of trans-local action and policy entrepreneurship provides additional explanatory power as these factors may strengthen favorable and, on some occasion, substitute unfavorable structural conditions.

Keywords Local Governance - Climate Policy · Trans-local Action · QCA · Diffusion of innovations

\section{Einleitung}

Lokale Klimapolitik ist in ein komplexes Mehrebenensystem eingebettet. Städte treten zum einen als Adressaten von Regulierungen, Anreizen und Informationen übergeordneter Ebenen auf. Zum anderen sind Städte nicht auf eine mehr oder weniger passive Implementation von Vorgaben höherer Ebenen beschränkt; vielmehr können sie auf Optionen des Mehrebenensystems strategisch reagieren und diese beeinflussen (Bulkeley und Betsill 2013). Entsprechend rückt die überlokale Dimension städtischer Klimapolitik in den Fokus wissenschaftlicher Betrachtung (Bulkeley et al. 2012; Busch 2015; Kern 2014; Kemmerzell und Tews 2014). Bisherige Studien verweisen auf positive Effekte überlokalen Handelns für lokale Politikinnovationen, beispielsweise durch den erleichterten Austausch von Informationen. GleichermaBen dienen überlokale Aktivitäten lokalen Akteuren aber auch als Instrument, um politische Anliegen stadtintern voranzutreiben und Unterstützung zu mobilisieren sowie extern zur kollektiven Interessenvertretung von Städten (Kemmerzell und Tews 2014).

Die meisten Untersuchungen behandeln überlokales Handeln als abhängige Variable und untersuchen die Bestimmungsfaktoren der Mitgliedschaft in Netzwerkorganisationen oder die Umsetzung von mit solchen Mitgliedschaften verbundenen Selbstverpflichtungen. Das Ausmaß überlokaler Aktivitäten wird entweder durch strukturelle und institutionelle Faktoren oder durch Handlungsmotive lokaler Akteure erklärt. Bislang fehlt allerdings eine breiter angelegte Untersuchung zum Einfluss solcher Aktivitäten auf materielle Policies. Der vorliegende Beitrag soll zur Schließung dieser Lücke beitragen. Dabei wendet er ein diffusionsorientiertes Verständnis von Politikinnovation an (Jordan und Huitema 2014) und untersucht die Wirkung überlokalen Handelns auf die Dichte bzw. Anzahl beschlossener innovativer Maßnahmen (Schaffrin et al. 2015) in deutschen Großstädten im Kontext lokaler politischer und sozioökonomischer Bedingungen. Die Fragestellung richtet sich also auf die Extensivität von Policies, nicht auf die Intensität der Maßnahmen. Zudem werden neben überlokalem Handeln auch andere Kontextfaktoren in die Analyse einbezogen. Um eine in vielen Studien übliche Tendenz des „everything matters“ zu vermeiden (Ryan 2015, S. 520), wählen wir eine qualitativ-komparative Untersuchungsstrategie, die nicht auf die Erklärung des Einflusses einzelner Variablen abzielt, sondern auf die Identifikation bestimmter Konfigurationen von Bedingungen, 
die innovative Klimapolitik erklären können (Ragin 1987; Schneider und Wagemann 2012).

Wir verstehen Klimapolitik im Sinne von Klimaschutz und fassen darunter Maßnahmen, die auf eine Vermeidung oder Abschwächung des Klimawandels durch die Reduktion von Treibhausgasemissionen abzielen. Für (Groß-)Städte sind vor allem Maßnahmen zur Steigerung der Energieeffizienz und zum Energiesparen von Bedeutung (Stuttgart 2016). Global fällt in Städten 60 bis $80 \%$ des Energiekonsums an, mit steigender Tendenz (UN Habitat 2011). Dies macht Städte zu zentralen Akteuren der Klimaschutzpolitik, da mit den zu realisierenden Einspar- und Effizienzpotenzialen ein wesentlicher Beitrag zur Reduktion der Treibhausgasemissionen geleistet werden kann. Die effiziente und sparsame Bereitstellung und Nutzung von Energie in Form von Wärme und Strom können dabei in unterschiedlichen Handlungsfeldern relevant sein, wie etwa in städtischen und privaten Immobilien, in Stadtplanungskonzepten und im Mobilitätsmanagement. Um diesem Umstand Rechnung zu tragen, wenden wir ein breites Verständnis städtischer Klimapolitik an und berücksichtigen Maßnahmen in verschiedenen Handlungsfeldern.

Im Folgenden wird zunächst der analytische Untersuchungsrahmen entwickelt. Dieser beruht auf der Annahme, dass lokalspezifische Bedingungen zwar einen bestimmten Handlungsspielraum vorgeben, in ihrer Wirkung jedoch nicht eindeutig sind. Innerhalb dieses strukturellen Rahmens bleibt ein Handlungsspielraum, den Akteure unter anderem durch überlokales Handeln in den vertikalen und horizontalen Strukturen des überlokalen Handlungsraums lokaler Politik ausfüllen können. Im darauffolgenden Abschnitt werden das Forschungsdesign, die Untersuchungsmethode (Qualitative Comparative Analysis - QCA) sowie die zugrundeliegenden Daten präsentiert. ${ }^{1}$ Die Ergebnisse der Untersuchung zeigen, dass strukturelle Faktoren die Extensivität von Klimaschutzmaßnahmen in Großstädten nur begrenzt erklären können. Die Berücksichtigung überlokalen Handelns und politischer Unternehmer führt zu einer erhöhten Erklärungskraft, da diese Faktoren günstige Rahmenbedingungen verstärken oder in einigen Fällen sogar eine substituierende Wirkung entfalten können.

\section{Forschungsstand und analytischer Rahmen}

Zunächst sollen Überlegungen zur Operationalisierung des Outcomes ${ }^{2}$ erfolgen. Wie kann eine innovative städtische Klimapolitik erfasst und gemessen werden? Andrew Jordan und David Huitema unterscheiden drei Dimensionen klimapolitischer Innovationen: ,invention“, ,diffusion“ und „ex post evaluation“ (Jordan und Huitema

\footnotetext{
1 Die Daten wurden im Rahmen des DFG-finanzierten Forschungsprojektes „Überlokales Handeln und lokale Innovationen im Klimawandel. Eine vergleichende Analyse deutscher Großstädte“ an der Technischen Universität Darmstadt erhoben und ausgewertet.

2 Aus methodischen Gründen verwenden wir entsprechend der QCA-Terminologie den Begriff „Outcome“ für die politischen Maßnahmen. In der vergleichenden Policy-Forschung würden diese üblicherweise als Output bezeichnet, während Outcome für weiterreichende Politikergebnisse steht. Um einer Begriffsverwirrung vorzubeugen, beschränken wir uns allerdings auf die Bezeichnung Outcome. Den Hinweis zur Begriffsklärung verdanken wir einem bzw. einer der GutachterInnen.
} 
2014, S. 717). Da es uns nicht um die Entwicklung neuer Ansätze im Klimaschutz geht (Invention), sondern die Übernahme von erfolgreichen Policies im Fokus steht, konzentrieren wir uns auf die Dimension der Diffusion von „Good Practices“. Diese Perspektive ist verbunden mit Policy Transfer, Lernen und Lesson-Drawing sowie der Adaption von Policies an lokale Bedingungen. Dafür ist eine Messung lokaler Klimaschutzpolitik notwendig. In der Literatur zu Policy Diffusion (Holzinger et al. 2007) wird grundlegend zwischen einem „density“ und einem ,intensity approach“ unterschieden (Knill et al. 2012; Schaffrin et al. 2015). Der Ansatz der vorliegenden Untersuchung und die mittlere bis große Fallzahl legen die Anwendung des „density approach“ nahe, der im Wesentlichen nach der Dichte bzw. Anzahl innovativer Politikinstrumente in einem Feld fragt. Eine Analyse der Intensität gemäß des „,intensity approach“ würde hingegen die Berücksichtigung der Reichweite von Instrumenten, ihrer Finanzierungsbasis sowie ortsspezifischen Anwendung erfordern, was im Rahmen von Fallstudien sinnvoller bearbeitet werden kann (zu den Details des Outcomes siehe Abschn. 3.3). Politikinnovation wird also verstanden als die Diffusion von Politikinstrumenten im Feld lokaler Klimaschutzpolitik, gemessen an der Extensivität beschlossener Instrumente.

Erfolgsbedingungen lokaler Klimapolitik stehen im Fokus von Studien aus unterschiedlichen Blickwinkeln. Zumeist werden einzelne Faktoren und ihr Einfluss auf lokale Klimapolitik isoliert betrachtet. Zu diesen Faktoren zählen, erstens, besondere naturräumliche und sozioökonomische Gegebenheiten einer Stadt, die eine erhöhte Betroffenheit von den Folgen des Klimawandels begründen. Damit sind zum Beispiel die physische Betroffenheit einer Stadt durch den Klimawandel, aber auch bestimmte langfristige und damit quasi naturwüchsige infrastrukturelle oder sozioökonomische Bedingungen gemeint. In der Literatur zum kommunalen Klimaschutz werden solche Bedingungen als „climate risk“ (Gefährdung durch den Klimawandel) oder „climate stress“ (Beitrag zum Klimawandel, z.B. durch einen hohen Beschäftigungsanteil im industriellen Sektor) erfasst (Zahran et al. 2008; Krause 2013).

Des Weiteren müssen die politischen Machtverhältnisse, die wirtschaftliche Situation der Kommune und der Stadtbevölkerung sowie administrative Kapazitäten berücksichtigt werden. Solche Bedingungen können sowohl aus Ansätzen der vergleichenden Staatstätigkeitsforschung (Zohlnhöfer 2008) als auch aus Analysen zum lokalen Klimaschutz abgeleitet werden. Relevante Bedingungen werden zunächst mithilfe von „,demand-side“-Indikatoren operationalisiert. Die Nachfrage nach ökologisch orientierter Politik wird zum Beispiel durch politische Mehrheitsverhältnisse, zivilgesellschaftliche Aktivitäten, ein hohes Bildungsniveau oder ein hohes durchschnittliches Haushaltseinkommen (Krause 2011; Pitt 2010; Sharp et al. 2011) beeinflusst. Auf der "supply-side“ sind Indikatoren wie administrative Kapazitäten, die Zusammensetzung von Regierungen (Schmidt 1996; Knill et al. 2010) oder die finanziellen Möglichkeiten und Restriktionen einer Kommune verortet (Sharp et al. 2011; Homsy und Warner 2015). Krause (2011) erwähnt zudem die Bedeutung von „policy champions“ als Entrepreneure einer innovativen Klimaschutzpolitik. Deren Rolle betonen auch Mintrom und Luetjens (2017) sowie Jordan und Huitema (2014, S. 723) mit Verweis auf die Diffusion und Implementierung von Policy-Innovationen. 
Die vergleichende Staatstätigkeitsforschung unterstreicht die zunehmende Bedeutung der Internationalisierung von Politik (Zohlnhöfer 2008, S. 161). Auf kommunaler Ebene wird diese durch die Einbindung von Städten in Strukturen der Multilevel Governance (MLG) (Kern 2014) und die damit verbundenen Optionen überlokalen Handelns (Kemmerzell und Tews 2014) repräsentiert. So konnten sowohl der Einfluss horizontaler Strukturen (z.B. Mitgliedschaft in energie- und klimapolitischen Netzwerken) auf die Wahrscheinlichkeit der Verabschiedung eines integrierten Klimaschutzkonzeptes (Hakelberg 2014) als auch die Bedeutung von vertikalen Aspekten der MLG (Interaktionen über Regierungsebenen hinweg, ob über Konsultationen oder die Bereitstellung von Fördergeldern) für nachhaltige Energie- und Klimaschutzkonzepte auf lokaler Ebene (Homsy und Warner 2015) gezeigt werden. Eine analytische Trennung der horizontalen und vertikalen Dimension erscheint hier angebracht, da jeweils unterschiedliche Dynamiken präsent sind. Aktivitäten in der vertikalen Dimension basieren zumeist auf Regelungen und Anreizen von höheren Ebenen. Lokale Akteure reagieren auf Vorgaben und Angebote und integrieren diese in lokale Politikprozesse. Dabei verlaufen die Interaktionsprozesse tendenziell unidirektional. Kommunen nehmen Fördergelder entgegen oder vermitteln Interessen nach oben weiter. Aktivitäten in der horizontalen Dimension sind eher von einem gegenseitigen Informationsaustausch ,,auf Augenhöhe“ geprägt.

Bisherige Forschung hat jedoch gezeigt, dass lokale Akteure die horizontale und vertikale Dimension überlokalen Handelns als einheitlichen Handlungsraum wahrnehmen. Aktivitäten in beiden Dimensionen gehen ineinander über oder bedingen sich gegenseitig. So kann beispielsweise die Mitgliedschaft in Städtenetzwerken auch der Interessenvermittlung oder der Rekrutierung von Projektpartnern für die Beantragung von Fördermitteln dienen. In der täglichen Verwaltungsarbeit wird nicht zwischen unterschiedlichen Arten von Aktivitäten, die über die Stadtgrenzen hinausgehen, differenziert. Daher bietet es sich an, von einem überlokalen Handlungsraum zu sprechen, um beide Dimensionen integriert zu betrachten (Kemmerzell und Tews 2014). Überlokale Aktivitäten in beiden Dimensionen erweitern den Handlungsspielraum lokaler Akteure, da ihnen neue Instrumente zur Verfolgung ihrer politischen Ziele an die Hand gegeben werden.

Ein Manko der bestehenden Forschung zu städtischer Klimapolitik liegt darin, dass der Einfluss der unterschiedlichen Erfolgsfaktoren zumeist nur isoliert betrachtet wird bzw. die Erklärungskraft der Faktoren gegenübergestellt wird oder eine Tendenz des ,everything matters“ besteht (Ryan 2015, S. 520). Wir streben hingegen ein integriertes konfiguratives Modell zur Erklärung innovativer lokaler Klimapolitik an, ,to identify and analyze different and alternative configurations of conditions that affect [...] urban climate policy“ (Ryan 2015, S. 520). „Konfigurativ“ meint in diesem Zusammenhang, dass bestimmte Erfolgsfaktoren ihre Wirkung im Zusammenspiel mit anderen Faktoren entfalten. Der kurze Überblick über mögliche Erfolgsfaktoren verweist auf zwei Gruppen von Bedingungen: Bei den „Kontextfaktoren“ handelt es sich weitgehend um strukturelle Bedingungen, die über einen längeren Zeitraum stabil und kurzfristiger politischer Veränderbarkeit zumeist nicht zugänglich sind. Damit können sie als „distant to the outcome“ (Schneider und Wagemann 2012) bezeichnet werden. „Close to the outcome“ (Schneider und Wagemann 2012) sind hingegen solche Faktoren, die sich unmittelbar auf die „Handlungen lokaler Ak- 
teure" beziehen und sich durch ein höheres Maß an Beeinflussbarkeit auszeichnen. Dazu gehören die beiden Dimensionen überlokalen Handelns, aber auch ,,policy entrepreneurship“.

\section{Datenbasis, Forschungsdesign und Hypothesen}

Um die Frage nach den Bedingungen für eine innovative bzw. extensive städtische Klimapolitik zu beantworten und dabei die bereits genannte methodische Falle des „everything matters“ zu vermeiden, verwenden wir ein zweistufiges Untersuchungsdesign mit einem qualitativ-komparativen Untersuchungsansatz. Dieser zielt nicht auf die Erklärung des Einflusses einzelner Variablen, sondern auf die Identifikation bestimmter Konfigurationen von Bedingungen, die innovative Klimapolitik erklären können. Im Folgenden werden die Fallauswahl und Datenbasis der Untersuchung erläutert, bevor das Forschungsdesign und die gewählte Methode näher beschrieben werden.

\subsection{Datenbasis}

Zur Operationalisierung innovativer Klimapolitik und ihrer Erfolgsbedingungen wurden Daten aus unterschiedlichen Quellen erhoben. Die Daten wurden zum Teil aus Statistiken der Städte, der Länder, von Städtenetzwerken sowie der Bundesagentur für Arbeit gewonnen und sind online verfügbar (vgl. Abschn. 3.3.1 und Online-Anhang). Weitere Daten wurden mithilfe einer Umfrage erhoben, die im Frühjahr 2016 unter 85 deutschen Städten durchgeführt wurde. Der Fragebogen umfasst 14 Fragengruppen, die sowohl Einschätzungen städtischer Akteure als auch Fakten zum Stand der städtischen Klimapolitik abfragen. Die Fragebögen wurden an die Büros der OberbürgermeisterInnen mit der Bitte versandt, diese an die zuständige Stelle in der Stadtverwaltung weiterzuleiten. Die Daten gehen somit auf die Aussagen von ExpertInnen der Stadtverwaltung zurück.

Auswahlkriterium für die Fälle war, dass eine Stadt zwischen 1990 und 2013 mindestens bei einer Zählung den Großstadtstatus besessen hat. ${ }^{3}$ Daher umfasst das Gesamtsample 85 Städte, obwohl 2013 nur 77 Städte als Großstadt gezählt werden konnten. Die Fallauswahl beruht auf der ,,comparable cases strategy“ (Lijphart 1975) und verwendet deshalb ein nicht-randomisiertes Sample. Die Beschränkung auf Großstädte lässt sich damit erklären, dass ein sinnvoller Vergleich der Maßnahmendichte das Vorhandensein bestimmter Schlüsselfaktoren, etwa eine von hauptberuflichen politischen Beamten geleitete, funktional differenzierte Verwaltung, voraussetzt.

\footnotetext{
3 Gemäß der Definition des Bundesinstituts für Bau-, Stadt- und Raumforschung gelten Städte mit über 100.000 EinwohnerInnen als Großstadt.
} 
Tab. 1 Vergleich Gesamtsample und Rücklauf

\begin{tabular}{llll}
\hline & $\begin{array}{l}\text { Großstädte } \\
(N=85)\end{array}$ & $\begin{array}{l}\text { Teilnehmer an } \\
\text { Umfrage }(N=71)\end{array}$ & $\begin{array}{l}\text { Rücklauf } \\
(\%)\end{array}$ \\
\hline Bevölkerung (2013) über 1.000.000 & 4 & 4 & 100 \\
Bevölkerung 500.000-1.000.000 & 9 & 9 & 100 \\
Bevölkerung 200.000-500.000 & 26 & 21 & 81 \\
Bevölkerung unter 200.000 & 46 & 37 & 80 \\
Durchschnittliche Einwohnerzahl & 304.980 & 331.267 & - \\
Stadtstaat & 3 & 3 & 100 \\
Kreisfreie Stadt & 68 & 56 & 82 \\
Kreisangehörige Stadt & 11 & 9 & 82 \\
Spezieller Status: Metropolregion & 3 & 3 & 100 \\
Lage: Alte Bundesländer & 73 & 61 & 84 \\
Lage: Neue Bundesländer & 12 & 10 & 83 \\
Medianeinkommen pro Kopf $(€, 2012)$ & 3079 & 3090 & - \\
Beschäftigung im tertiären Sektor $(\%, 2012)$ & 79,4 & 79,7 & - \\
Verschuldung pro Kopf $(€$, 2014) & 3900 & 4198 & - \\
Steuereinnahmekraft pro Kopf $(€, 2014)$ & 1212 & 1243 & - \\
\hline
\end{tabular}

Von den 85 angeschriebenen Städten beteiligten sich 71 an der Umfrage, dies entspricht einer Rücklaufquote von $84 \%$. Während die Rückläufe hinsichtlich der Größenklassen von Städten nicht vollständig gleichmäßig verteilt sind ${ }^{4}$, ergeben sich keine statistischen Auffälligkeiten hinsichtlich weiterer Basisgrößen, wie Medianeinkommen, Steuereinnahmekraft, Verschuldung, Beschäftigungsstruktur oder geografische Lage (siehe Tab. 1).

\subsection{Forschungsdesign und Methode}

Die Analyse verfolgt eine qualitativ-komparative Untersuchungsstrategie, die nicht auf die Erklärung des Einflusses einzelner Variablen abzielt, sondern auf Konfigurationen von Bedingungen. Ein solches Vorgehen, verbunden mit einem theoretisch ausgewählten Sample, impliziert einen ,causes-of-effects“-Ansatz, der im Gegensatz zu quasi-experimentellen Designs des „effects-of-causes“-Ansatzes steht (Goertz und Mahoney 2012). Damit zielt der Beitrag auf die Identifikation hinreichender und/oder notwendiger Bedingungen zur Erklärung eines Outcomes. Aufgrund der relativ großen Fallzahl und dem Interesse an verallgemeinernden Schlussfolgerungen erscheint ein qualitativ-idiografisches Verfahren nicht geeignet. Daher verwenden wir die mengentheoretische Methode der Qualitativ-komparativen Analyse (QCA), die einerseits eine Beibehaltung der fallorientierten Logik, andererseits aber auch die Abbildung von Kausalität und das Ziehen von Schlussfolgerungen ermöglicht (Schneider und Wagemann 2012). In der Policy-Forschung hat QCA in den vergangenen Jahren vermehrte Anwendung gefunden. Wichtige Studien sind hierbei

\footnotetext{
${ }^{4}$ Es haben sich alle Städte mit über 500.000 EinwohnerInnen beteiligt, unter den kleinen Großstädten ist die Nichtbeteiligung gleichmäßig verteilt.
} 
unter anderem die Analyse von Arbeitsmarktregimen durch Emmenegger (2011), die Untersuchung nationaler Energieeffizienzpolitik durch Mayer et al. (2011), die Beiträge von Hörisch (2013) zur Fiskalpolitik im Rahmen der europäischen Finanzkrise sowie von Stoiber und Töller (2016) zur Privatisierung des Maßregelvollzugs im Bundesländervergleich. Eine Anwendung auf den Gegenstandsbereich lokaler Klimapolitik steht jedoch noch aus. ${ }^{5}$

In der klassischen Variante beruht QCA auf der Anwendung boolescher Algebra und der damit verbundenen Dichotomisierung von Daten. Ein Fall (in dieser Untersuchung: eine Stadt) wird als Konfiguration mehrerer Merkmale dargestellt, die einen bestimmten Outcome aufweist. Wissenschaftstheoretisch ist QCA im Feld der formalen Logik verortet (Siewert 2017, S. 274), die Aussagen die Wahrheitswerte Wahr (1) oder Falsch (0) zuordnet. Notwendige Voraussetzung einer QCA ist die Kalibrierung bzw. Kodierung von Bedingungen, also die Zuweisung von Wahrheitswerten. Während in der klassischen Variante jeweils die Werte 1 und 0 zugeordnet werden (Crisp Set QCA), erlauben weitere Varianten der QCA die Zuordnung mehrerer Werte (Multi Value QCA) oder eines Wertes zwischen 0 und 1, um eine abgestufte Zugehörigkeit zu einer Menge abzubilden (Fuzzy Set QCA).

Wie bereits erwähnt, konzentriert sich dieser Artikel auf die Identifikation von Fallgruppen, weshalb wir die klassische Variante der Crisp Set QCA anwenden. Der durch die Dichotomisierung bewirkte Verlust an Informationen wird u. E. durch zwei Vorzüge aufgewogen: erstens durch die vergleichsweise höhere Trennschärfe des Verfahrens und zweitens durch eine klarere Interpretierbarkeit des Abdeckungsparameters, der für unser Modell eine entscheidende Rolle spielt. Zudem wenden wir ein konservatives Verfahren an und lassen nur solche Konfigurationen als „wahr“ gelten, die einen Konsistenzwert von 1,0 erreichen. Mit dem Parameter der Abdeckung können wir die Anzahl der durch das Modell erklärten Fälle bestimmen. Angesichts der strikten Anwendung des Konsistenzkriteriums rückt die Abdeckung damit in den Vordergrund bei der Interpretation der Analyse. ${ }^{6}$

Wir wenden QCA in einem Zwei-Schritt-Verfahren an (Schneider und Wagemann 2012, S. 253; Mayer et al. 2011) und unterteilen die zu untersuchenden Bedingungen in „ferne“ und „,nahe“ Faktoren. Ferne Faktoren sind relativ stabil und von den handelnden Akteuren nicht oder kaum veränderbar. Der Einfluss auf den Outcome ist eher indirekt (Mayer et al. 2011, S. 405). Nahe Faktoren sind leichter und somit auch gezielt beeinflussbar. Sie wirken zudem direkter auf den Outcome (Mayer et al. 2011, S. 405). Wir erwarten, dass durch Hinzunahme der nahen Faktoren kontradiktorische Konfigurationen, also solche, in denen der Outcome sowohl die Ausprägungen 1 als auch 0 annimmt, aufgelöst werden und die Abdeckung des gesamten Erklärungsmodells erhöht wird. Dabei erfolgt eine Prüfung, inwie-

\footnotetext{
5 Für eine detaillierte Beschreibung der Grundlagen von QCA siehe Ragin (1987), Berg-Schlosser und Cronqvist (2012), Schneider und Wagemann (2012), Legewie (2013).

6 Folgendes Beispiel sei zur Veranschaulichung der Konzepte Konsistenz und Abdeckung gegeben: 19 von 20 Fällen einer hypothetischen Konfiguration besitzen den Outcome 1, einer den Outcome 0. Die Konsistenz der Aussage, dass diese Konfiguration den Outcome 1 produziert, ist also 0,95 (19 dividiert durch 20). Wenn nun insgesamt 50 Fälle den Outcome 1 besitzen, beträgt die Abdeckung der Konfiguration 0,38 (19 dividiert durch 50).
} 
fern die beiden Dimensionen überlokalen Handelns als INUS-Bedingungen ${ }^{7}$ einen eigenständigen Beitrag zur Erklärung leisten können. Die einzelnen Bedingungen, deren Zusammenfassung in einem allgemeinen Modell sowie die zu überprüfenden Hypothesen werden im folgenden Kapitel näher ausgeführt.

\subsection{Outcome, Bedingungen und Hypothesen}

Zunächst wird die Auswahl und Operationalisierung der Untersuchungsgrößen dargestellt. Wir beginnen mit der Beschreibung des Outcomes. Die Bedingungen unterteilen wir im Sinne unseres Forschungsdesigns in ferne und nahe Faktoren. Anschließend verknüpfen wir Outcome und Bedingungen durch drei Hypothesensets.

\subsubsection{Outcome: Extensivität von Klimaschutzmaßnahmen}

Der Outcome der Untersuchung besteht in der Extensivität von Politikinnovationen im Bereich Klimaschutz und wurde im Fragebogen durch eine Liste von 33 beschlossenen Maßnahmen abgefragt (siehe Tab. 2). Der informationelle Charakter dieser Faktenabfrage ermöglicht im Prinzip die Kontrolle möglicher falscher oder Tendenzangaben, was an einigen Stellen auch durchgeführt wurde (vergleiche Krause 2011, S. 53-54). Die Erstellung der Liste beruht auf unterschiedlichen Quellen. Erstens wurde die relevante Literatur zum Klimaschutz in deutschen Kommunen für einen ersten Überblick herangezogen (Kern et al. 2005; Bulkeley und Kern 2006; Difu 2013). Zweitens konnte auf die Erkenntnisse aus einem bereits abgeschlossenen Projekt zurückgegriffen werden, das den Umgang mit dem Klimawandel in drei deutschen Städten (Frankfurt, München und Stuttgart) untersuchte. Drittens wurde ein Pretest des Fragebogens in Städten durchgeführt, die nicht in der Fallauswahl enthalten sind.

Die Instrumente des Outcomes werden folgenden Kategorien zugeordnet: (1) Implementation und Evaluation von Maßnahmen; (2) ,self-governing“, dies umfasst Maßnahmen innerhalb kommunaler Liegenschaften; (3) Maßnahmen, die Stadtplanung und private Liegenschaften betreffen; (4) Transport und Verkehr; (5) „weiche“ Maßnahmen, die insbesondere Beratung und Informationen umfassen. Die einzelnen Maßnahmengruppen gehen gleichgewichtet (also jeweils zu 20\%) in einen Index innovativer Policies ein. Die Gewichtung geht auf die theoretische Überlegung zurück, dass die Indexierung ein breites Spektrum von Maßnahmen berücksichtigt und die einzelnen Maßnahmen eine unterschiedliche qualitative Bedeutung besitzen (dies gilt insbesondere für $\mathrm{CO}_{2}$-Bilanzierung und Monitoring). Zur Indexbildung werden in jeder Kategorie die Anzahl der beschlossenen durch die Anzahl der möglichen Maßnahmen dividiert, die Summe der Einzelwerte wird schließlich durch fünf geteilt. Damit ergibt sich ein theoretisch möglicher Indexwert zwischen 0 und 1. Der so gewonnene Index innovativer Maßnahmen besitzt in der Empirie eine Spannbreite von 0,19 bis 0,96. Die grafische Darstellung (siehe Abb. 1) weist trotz der recht kontinuierlichen Verteilung eine auffällige Teilung in zwei Cluster beim Indexwert

\footnotetext{
7 INUS (,insufficient, but necessary part of an unnecessary but sufficient condition“) bezeichnet solche Bedingungen, die in Konjunktion Bestandteil einer Lösung sind, die aus mehreren Lösungstermen besteht.
} 
Tab. 2 Kommunale Klimaschutzmaßnahmen

Implementation und Evaluation

1) regelmäßige Erstellung einer $\mathrm{CO}_{2}$-Bilanz

2) regelmäßiges Monitoring des Energieverbrauchs

Self-Governing

3) Nutzung städtischer Gebäude/Flächen zur Erzeugung erneuerbarer Energien

4) Beschaffung energieeffizienter oder emissionsarmer Fahrzeuge

5) Nutzung von Kraft-Wärme-Kopplung zur Versorgung städtischer Gebäude

6) Beschaffung energieeffizienter Bürogeräte

7) Neubau und Sanierung von städtischen Gebäuden über Standards der EnEV hinaus

8) stadtinternes „Contracting“ zur Finanzierung städtischer Energiesparmaßnahmen

9) „Contracting“ mit Privaten zur Finanzierung städtischer Energiesparmaßnahmen

10) Ausbau des Anteils erneuerbarer Energien an der städtischen Energieversorgung

11) Divestment

Stadtplanung und private Immobilien

12) Benennung von Modellstadtbezirken für Klimaschutzmaßnahmen

13) energiepolitische Festsetzungen in Bebauungsplänen oder städtebaulichen Verträgen

14) spezielle Satzungen (z. B. Anschluss- und Benutzungszwang für Fernwärme)

15) Erhalt und Entwicklung von Grünflächen und Grünzügen

16) Ermittlung des Potenzials für Solaranlagen im Stadtgebiet (z. B. Solarkataster)

17) Förderung von Niedrigenergie- oder Passivhäusern

18) Förderung der Nutzung klimafreundlicher Technik in privaten Neubauten und Bestand

19) Förderung der energetischen Sanierung privater Gebäude über EnEV hinaus

Verkehr

20) energieeffiziente Straßenbeleuchtung

21) Maßnahmen zur Steigerung der Attraktivität des ÖPNV

22) Nutzung emissionsarmer und energieeffizienter Fahrzeuge im ÖPNV

23) Förderung des Radverkehrs (Radwegeausbau, Einrichtung von Stellplätzen im Stadtgebiet)

24) Mobilitätsberatung, Mobilitätsmanagement

25) Förderung von Elektromobilität

Beratung, Information und Anreize

26) Förderung von Investitionen von Energieversorgern in Erzeugung regenerativer Energien

27) Unterstützung von Bürger-Energiegenossenschaften

28) Zuschüsse beim Kauf energieeffizienter Haushaltsgeräte für Privatpersonen

29) städtischer Fonds für private Klimaschutzprojekte

30) Öffentlichkeitsarbeit

31) Bewusstseinsbildung für klima- und ressourcenschonendes Verhalten

32) Beratung von Investoren und Unternehmen

33) Energieberatung für private Haushalte 


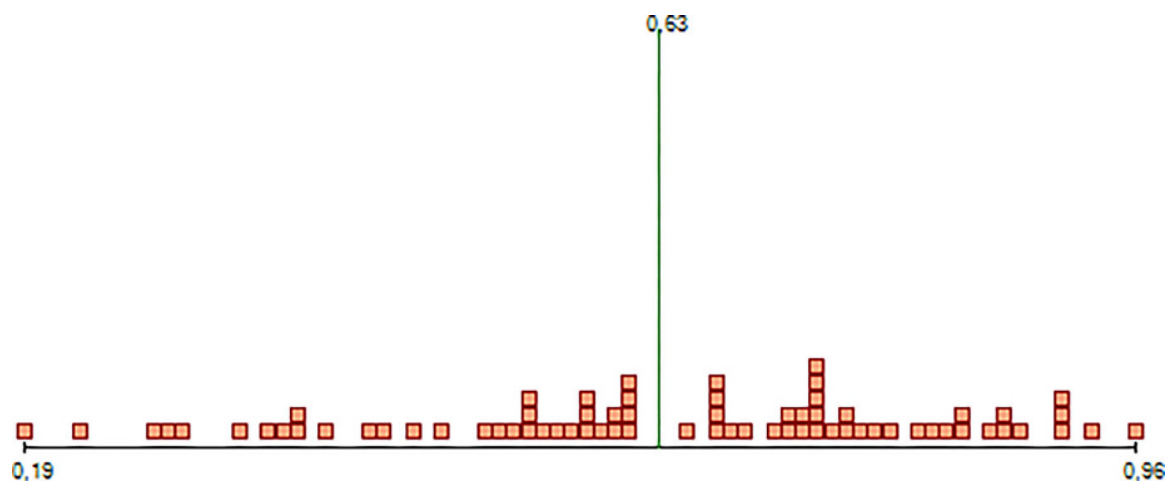

Abb. 1 Outcome - Kalibrierung (erstellt mit TOSMANA 1.5.2)

0,63 auf. Dieser Indexwert dient als Trennwert für die Kodierung des Outcomes mit 0 oder 1 . Dadurch werden 37 Städte mit dem Wert 1 und 34 Städte mit dem Wert 0 kodiert. ${ }^{8}$

\subsubsection{Ferne Faktoren}

Die vergleichende Analyse der Staatstätigkeitsforschung verweist zwar auf ein Set möglicher Bedingungen, diese sind allerdings abstrakt und müssen im Hinblick auf die vorliegende Fragestellung eingegrenzt und konkretisiert werden. Wir haben die Bedingungen gemäß ihrer theoretischen Rückbindung (vgl. Abschn. 2) und ihrer Anwendbarkeit auf den Betrachtungsgegenstand „Großstadt“ ausgewählt und operationalisiert: Bevölkerungsgröße, wirtschaftliche Situation, Stärke der Grünen und anderer ökologischer Parteien sowie Problembetroffenheit und Problembewusstsein.

Bevölkerungsgröße (Abkürzung: $B$ ) dient als Proxy für generelle administrative Kapazitäten einer Stadt, da größere Städte über mehr verteilbare Ressourcen und mehr Beschäftigte verfügen. Dies ist insofern wichtig, da die erhobenen Maßnahmen zum größten Teil nicht in den Bereich kommunaler Pflichtaufgaben fallen. Städte mit größeren administrativen Kapazitäten können eine extensivere und innovativere Klimaschutzpolitik betreiben. Die Bedingung wurde mithilfe der durch die Kommunale Gemeinschaftsstelle für Verwaltungsmanagement (KGST) vorgenommenen Einteilung von Städten in Größenklassen kalibriert. Städte der Größenklassen 1 und 2 (ab 200.000 Einwohner) werden mit dem Wert 1, Städte der Größenklassen 3 und 4 (unter 200.000 Einwohner) mit 0 kodiert. $^{9}$

\footnotetext{
8 Da es nicht unser Ziel ist, ein „Städteranking“ zu erstellen und wir dies den befragten Städten auch zugesagt haben, verzichten wir in diesem Artikel auf die Nennung von Städtenamen. Zwar werden alle Daten dokumentiert, durch „Anonymisierung“ ist ein Rückschluss auf einzelne Städte jedoch nicht möglich.

9 Online verfügbar unter: www.haushaltssteuerung.de/lexikon-einwohner-groessenklassen-vonkommunen.html. Zugegriffen: 10. Oktober 2018; siehe Online-Anhang für detaillierte Informationen zur Kalibrierung der Bedingungen und zu den Quellenangaben.
} 
Die wirtschaftliche Situation einer Kommune (Abkürzung: $W$ ) wird in der Literatur durch unterschiedliche Indikatoren operationalisiert. In einer bivariaten Korrelationsanalyse erwiesen sich Pro-Kopf-Einkommen und Steuereinnahmekraft als hoch signifikante Faktoren für Politikinnovationen. Beide Indikatoren beziehen sich allerdings auf unterschiedliche Grundlagen, zum einen auf die Stadtbevölkerung, zum anderen auf die verfügbaren Ressourcen der Gemeinde. Daher scheint es aus Gründen der analytischen Sparsamkeit angemessen, sie in einem Index zu integrieren, um die wirtschaftliche Situation einer Stadt abzubilden. Städte, die einen Indexwert annehmen, der oberhalb des Medians dieses Index liegt, werden mit 1 kodiert, alle anderen mit 0 .

Der Einfluss von Parteien und Mehrheitsverhältnissen auf politische Entscheidungen wird in der Forschung meist über die Parteiendifferenz gemessen, also als „supply-side“-Variable modelliert. In Bezug auf ökologische Themen müsste man im Sinne dieser Theorie, um die Stärke der Grünen und anderer ökologischer Parteien (Abkürzung: $G$ ) zu operationalisieren, die Anzahl grüner OberbürgermeisterInnen oder grüner DezernatsleiterInnen messen. Für die kommunale Ebene gestaltet sich diese Untersuchungsstrategie aufgrund der unterschiedlichen Kommunalverfassungen und aufgrund fehlender Parteimitgliedschaften in vielen Fällen allerdings schwierig. Daher haben wir entschieden, den Wahlerfolg grüner und anderer ökologischer Parteien als „,demand-side“-Faktor einzubeziehen. Dahinter steht die Überlegung, dass unabhängig von der Parteizugehörigkeit der politischen Beamten und der politischen Führung ein hoher Anteil grüner Wählerstimmen eine generelle Nachfrage nach ökologisch orientierten Policies signalisiert, an denen sich gewählte Politiker unabhängig von ihrer Parteizugehörigkeit orientieren müssen. Ein hoher Anteil grüner Wählerstimmen sollte somit zu einer innovativen Klimapolitik führen. In die Kalibrierung der Bedingung gehen sowohl die durchschnittlichen Wählerstimmen seit 1990 (arithmetisches Mittel) als auch der erstmalige Zeitpunkt eines überdurchschnittlichen Wahlerfolges ein. Städte, die im oberen Terzil der durchschnittlichen Stimmenanteile grüner Parteien oder im mittleren Terzil, verbunden mit einer frühzeitigen Stärke grüner/ökologischer Parteien (Wahlergebnis über dem arithmetischen Mittel bereits vor dem Jahr 2000), liegen, werden mit 1, alle anderen mit 0 kodiert.

Problembetroffenheit und Problembewusstsein (Abkürzung: $P$ ) bezieht sowohl geografische Faktoren als auch die allgemeine Problematisierung des Klimawandels ein, die im Fragebogen erhoben wurden. Wir gehen davon aus, dass Problembetroffenheit allein nicht ausreicht. Der Klimawandel und seine Folgen müssen in der Stadt auch aktiv thematisiert werden. An dieser Stelle könnte argumentiert werden, dass Betroffenheit durch den Klimawandel zwar Maßnahmen zur Anpassung an den Klimawandel erklären kann, nicht jedoch zum Klimaschutz. Die Auswahl dieser Bedingung beruht auf Forschungsergebnissen, die zeigen, dass die Konfrontation mit den Folgen des Klimawandels nicht nur zu Adaptionsmaßnahmen führt, sondern auch Klimaschutzpolicies befördern kann (vgl. Krause 2011, S. 50). In der Befragung nannten 61 der 71 Städte geografische Faktoren, die eine erhöhte Betroffenheit von den Folgen des Klimawandels der Stadt begründen. Zumeist betraf dies die Lage an Seen oder Flüssen (43 Städte) und eine hohe Bevölkerungsdichte (39). Die Lage an Gewässern führt zu einer stärkeren Betroffenheit von Hochwasser durch extremen Niederschlag, während eine hohe Siedlungsdichte anfälliger für Hitzepe- 
rioden macht, da eine Kühlung des Stadtgebiets erschwert wird. Dies wird durch eine Talkessellage (21 Städte) noch verschärft. 41 Städte sind von zwei oder mehr Risikofaktoren betroffen, was eine erhöhte Anfälligkeit für die Folgen des Klimawandels nahelegt. Zudem wird der Klimawandel in fast allen befragten Städten auch als Problem wahrgenommen und diskutiert (69 Städte), jedoch in unterschiedlichem Ausmaß. Auf die Frage, ob der Klimawandel in der Stadtpolitik und -verwaltung als Problem wahrgenommen wird, antworteten 38 Städte mit ,ja, sehr“ und 31 Städte mit ,ja, etwas“. Die Kalibrierung der Bedingung erfolgt anhand der beiden Kriterien „geografische Risikofaktoren“ und „Thematisierung des Klimawandels“. Falls dieser als großes Problem wahrgenommen wird oder eine mittlere Problemwahrnehmung in Verbindung mit dem Vorhandensein mindestens zweier Risikofaktoren angegeben wird, erfolgt eine Kodierung der Bedingung mit 1.

\subsubsection{Nahe Faktoren}

$\mathrm{Zu}$ den Faktoren, die näher am Outcome liegen und von den Akteuren leichter zu beeinflussen sind, zählen wir überlokale Aktivitäten einer Stadt und das Vorhandensein politischer Unternehmer, die Klimaschutzthemen über einen längeren Zeitraum vorantreiben.

Städtische Klimapolitik ist eingebettet in ein komplexes Mehrebenensystem (zum Konzept der Multilevel Governance siehe zum Beispiel Knodt und Große Hüttmann 2012), in dem der lokale Handlungsspielraum durch regulative Vorgaben, Angebote und Informationen von höheren Regierungsebenen eingeschränkt wird. Zugleich können lokale Akteure diesen Einfluss nutzen, um ihren Handlungsspielraum zu erweitern (Knodt 2010). Kristine Kern hat im Hinblick auf die städtische Ebene drei Governancemuster für die Steuerung von Klimapolitik im europäischen Mehrebenensystem identifiziert (Kern 2014). Hierarchische Governance umfasst dabei nur die zielgerichtete Steuerung städtischer Politik durch nationale oder supranationale Vorgaben, die von den Städten implementiert werden müssen. Vertikale Governance berücksichtigt die Wechselseitigkeit der Beziehungen zwischen den Ebenen. Die lokale Ebene reagiert zum einen auf Impulse von oben, wirkt zum anderen aber auch aktiv auf die Politikgestaltung auf höheren Ebenen ein. Horizontale Governance trägt schließlich der Beobachtung Rechnung, dass auch innerhalb einer Ebene Austauschbeziehungen bestehen können, die lokale Politikgestaltung beeinflussen. Hier sei exemplarisch auf transnationale Städtenetzwerke verwiesen (Kern 2014, S. 114-115). Hierarchische Governance spielt insgesamt eine untergeordnete Rolle, da es sich bei städtischer Klimaschutzpolitik, erstens, um eine weitgehend freiwillige Aufgabe handelt und, zweitens, kaum Variation regulativer Vorgaben im interkommunalen Vergleich besteht. Daher geht dieser Beitrag von zwei Dimensionen der Multilevel Governance aus, die Möglichkeiten zu überlokalen Aktivitäten bieten: eine vertikale und eine horizontale Dimension (Lee und Koski 2015, S. 1503-1505).

Überlokalem Handeln werden in beiden Dimensionen positive Effekte auf lokale Innovationen zugeschrieben, indem beispielsweise durch zusätzliche finanzielle Mittel und einen besseren Zugang zu ,best practices“ Policy-Lernen gefördert wird. Zudem können lokale Akteure auf Selbstverpflichtungen aus überlokalen Aktivitäten verweisen, damit Unterstützung und Teilhabe mobilisieren und die Umsetzung 


\section{Anzahl der Netzwerkmitgliedschaften}

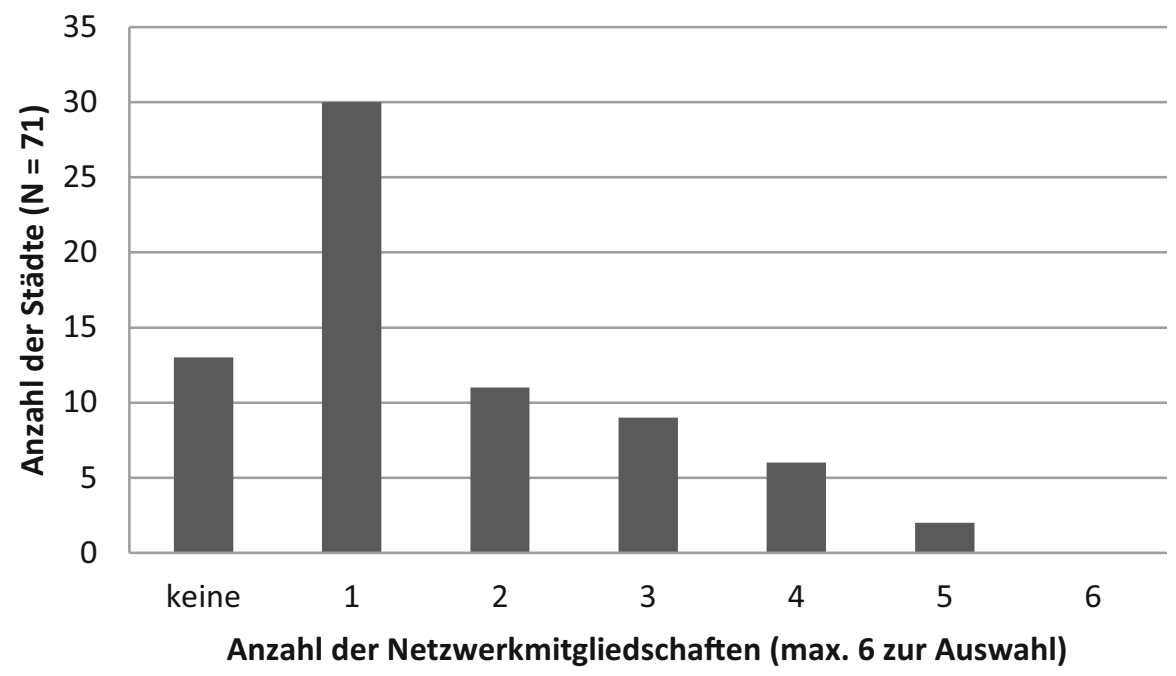

Abb. 2 Anzahl der Netzwerkmitgliedschaften (je Stadt)

klimapolitischer Maßnahmen vor Ort vorantreiben. Kostenintensive oder unbeliebte Maßnahmen können mit einem Verweis auf Fördermöglichkeiten, Selbstverpflichtungen in Städtenetzwerken etc. rechtfertigt werden. Hierbei ist zum Beispiel an die Erstellung von Energieaktionsplänen zu denken, die mit der Mitgliedschaft im Konvent der Bürgermeister (CoM) verbunden sind (Kemmerzell und Tews 2014; Bendlin 2016).

In der vertikalen Dimension überlokalen Handelns $(V)$ ist insbesondere die Akquirierung von Fördermitteln für lokale Akteure relevant. Die Mehrzahl der Städte hat in den letzten fünf Jahren Fördermittel aus Bundes- und Landesprojekten bezogen (67), zumeist aus ein bis vier Projekten. Nur vier Städte haben angegeben, keine Fördermittel erhalten zu haben. Projekte auf EU-Ebene sind deutlich weniger verbreitet. Lediglich 32 Städte führten in den letzten fünf Jahren EU-Projekte durch, meist nur ein bis zwei Projekte pro Stadt. Eine Kodierung der Bedingung mit 1 erfolgte, wenn Städte sich an beiden Formen vertikaler überlokaler Aktivitäten beteiligten oder eine hohe Beteiligungsfrequenz in einem Bereich aufweisen (Beteiligung an jeweils vier EU- oder Bundes- und Landesprojekten).

Die horizontale Dimension überlokalen Handelns $(H)$ umfasst die Mitgliedschaft in transnationalen Städtenetzwerken (Kern und Bulkeley 2009), die Teilnahme an Städtewettbewerben und Austauschbeziehungen mit anderen Städten außerhalb von Netzwerkbeziehungen. Wir konzentrieren uns auf das Engagement in Städtenetzwerken als langfristige und strategische Form der Kooperation. Die Mitgliedschaft in Städtenetzwerken und der regelmäßige Austausch mit Netzwerkmitgliedern sind wichtige Quellen für innovative Problemlösungen und Politikinstrumente. Die befragten Städte gaben Auskunft über die Intensität ihres Austauschs mit sechs ausgewählten themenspezifischen Netzwerken und deren Mitgliedern im Bereich Energie- 


\section{Anzahl der Netzwerke, zu denen Kontakt besteht}

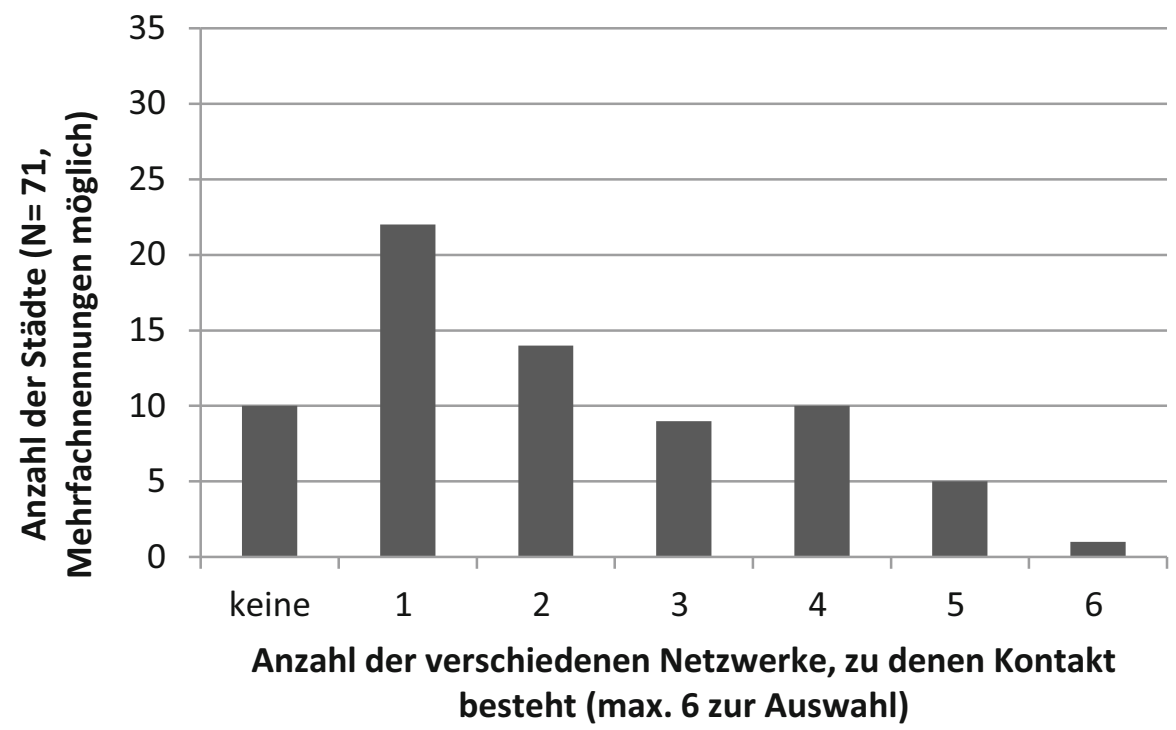

Abb. 3 Anzahl der unterschiedlichen Netzwerke, zu denen Kontakt besteht (je Stadt)

und Klimapolitik. Dazu zählten das Klima-Bündnis, das Netzwerk Energy Cities, der bereits erwähnte CoM, das Netzwerk Eurocities, ICLEI (Local governments for sustainability) und $C 40$ (Cities Climate Leadership Group).

Die Mehrzahl der 71 Städte in unserem Sample ist in mindestens einem der genannten Netzwerke formal Mitglied (58 von 71), 17 Städte davon sogar in mehr als zwei Netzwerken (siehe Abb. 2). Das Netzwerk mit der höchsten Mitgliedschaft (55 Mitglieder von 71) ist das Klima-Bündnis, gefolgt vom CoM mit 27 Mitgliedern. Diese Trends spiegeln sich auch in der Selbstauskunft der Städte zur Intensität ihrer Austauschbeziehungen mit diesen Netzwerken wider.

Die meisten Städte unterhalten Kontakte zum Klima-Bündnis (61) und zum CoM (29). Auch hier spielen pro Stadt tendenziell jeweils ein bis zwei Netzwerke eine Rolle. Nur sechs Städte pflegen Kontakte zu mehr als vier Netzwerken (siehe Abb. 3).

Interessanterweise decken sich die Mitgliedschaften nicht immer mit den tatsächlichen Austauschbeziehungen zu Klimaschutzthemen. Zum einen können Mitgliedschaften zu Netzwerken bestehen, ohne dass tatsächlich ein Kontakt stattfindet und ein Einfluss auf lokale Politik zu erwarten ist. Zum anderen wurden Austauschbeziehungen zu Netzwerken angegeben, in denen die jeweilige Stadt kein formales Mitglied ist. So gibt es beispielsweise sechs Städte, die Kontakte zu fünf oder sechs Netzwerken pflegen, aber nur zwei Städte, die formales Mitglied in jeweils fünf Netzwerken sind, und keine Stadt, die Mitglied in allen sechs Netzwerken ist. Dies deutet einerseits darauf hin, dass eine formale Mitgliedschaft nicht mit Kontakt und Austauschbeziehungen gleichzusetzen ist. Andererseits unterstreicht es die hohe Bedeutung von Netzwerken, die auch außerhalb einer formalen Mitgliedschaft 


\section{Aus welchem Bereich stammen politische Unternehmer?}

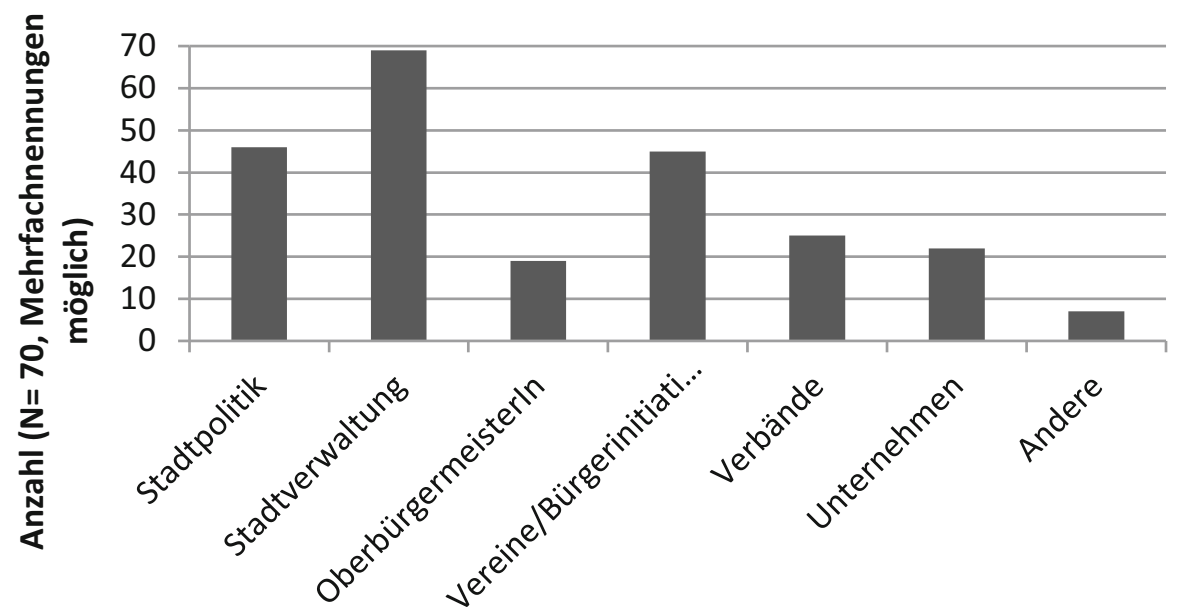

Abb. 4 Politische Unternehmer, die das Thema Klimaschutz kontinuierlich vorangetrieben haben

eine Rolle spielen. Wir beziehen daher nicht die formale Mitgliedschaft, sondern die Selbstauskunft zu den Austauschbeziehungen zwischen Städten und Netzwerken in die Analyse ein. Einige Städte erfüllen zudem besondere Aufgaben in diesen Netzwerken, die über eine einfache Mitgliedschaft bzw. Austauschbeziehungen hinausgehen. Dazu zählen die Leitung von Arbeitsgruppen, die Ausrichtung von Veranstaltungen und die Übernahme von Leitungsfunktionen in der Netzwerkorganisation, um nur einige Beispiele zu nennen. Auch hier zeigt sich, dass das KlimaBündnis eine besondere Rolle spielt. Vertreter aus 22 Städten üben hier eine besondere Funktion aus, während sich in den anderen Netzwerken mit jeweils zwei bis sieben Städten deutlich weniger Städte engagieren.

Damit beide Aspekte der Intensität horizontaler Aktivitäten berücksichtigt werden können, wurde zunächst ein Index gebildet, der die Anzahl der Netzwerke, zu denen Kontakt besteht, und besonderes Engagement (Leitungsfunktionen in Netzwerken oder Ausrichtung von Veranstaltungen) einbezieht. Beide Faktoren müssen jeweils überdurchschnittlich sein, also über dem arithmetischen Mittel aller Städte liegen. Damit ergibt sich eine Kodierung der Bedingung $\mathrm{H}$ mit 1, wenn Kontakt zu mehr als zwei Netzwerken und ein besonderes Engagement in mindestens einem Netzwerk vorliegen.

Als weiteren nahen Faktor beziehen wir neben überlokalem Handeln auch politisches Unternehmertum $(U)$ als akteursorientierten Erklärungsfaktor in die Analyse ein. In fast allen Städten (70 von 71) wurden Personen genannt, die das Thema Klimaschutz über mehrere Jahre hinweg kontinuierlich vorangetrieben haben (siehe Abb. 4). Meist stammen diese Personen aus der Stadtverwaltung (69). Jedoch wurden auch Personen aus der Stadtpolitik (46) und der Zivilgesellschaft in Form von Vereinen und Bürgerinitiativen (45 Nennungen) genannt. OberbürgermeisterInnen (OB) wurden in geringerem Maße genannt (19 Nennungen). Aus vorangegangenen 


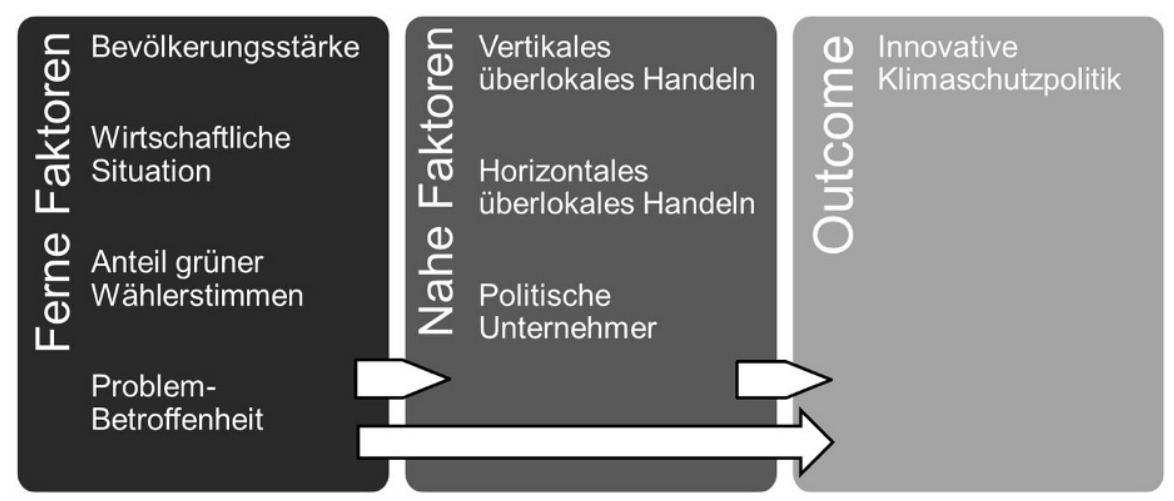

Abb. 5 Wirkungsmodell der Kausalfaktoren

Studien ist jedoch bekannt, dass die Unterstützung durch die politische Führung, und hier besonders durch den Oberbürgermeister bzw. die Oberbürgermeisterin, einen wichtigen Faktor für die Spielräume der Fachverwaltungen darstellt (Gissendanner 2002). Das besondere Engagement von OberbürgermeisterInnen für den Klimaschutz fördert innovative Klimaschutzpolitik (Barber 2013), da so eine strategische Unterstützung der stadtweiten Klimaschutzpolitik signalisiert wird, die eine Planung und Implementierung von Maßnahmen erleichtert.

Da fast alle Städte politische Unternehmer identifiziert haben, konzentrieren wir uns bei der Kalibrierung der Bedingung daher nicht auf die Existenz von politischen Unternehmern, sondern auf Fälle mit engagierten OberbürgermeisterInnen (OB). Falls eine oder ein $\mathrm{OB}$ als politscher Unternehmer genannt wurde, erfolgt eine Kodierung mit dem Wert $1 .^{10}$

\subsubsection{Hypothesen}

Zur Strukturierung der nachfolgenden Analyse werden drei Hypothesengruppen gebildet, welche die beiden Faktorenbündel verbinden. Die Hypothesen basieren auf den theoretischen Vorüberlegungen in den Abschn. 3.3.2 und 3.3.3. Wir gehen davon aus, dass die fernen Faktoren politische Entscheidungen prägen, aber nicht determinieren (siehe Abb. 5). Daher sollte der Einbezug naher Faktoren die Erklärungskraft der theoretischen Modelle erhöhen bzw. nicht erklärte Widersprüche auflösen.

Hypothesen $1 a$ und $1 b$ beziehen sich ausschließlich auf die fernen, strukturellen Faktoren:

H1a Das gemeinsame Vorliegen aller strukturellen Bedingungen (Ausprägung jeweils 1) ist hinreichend für eine hohe Extensivität lokaler Klimaschutzmaßnahmen.

10 Siehe Online-Anhang für den kodierten Komplettdatensatz. 
H1b Die gemeinsame Abwesenheit aller strukturellen Bedingungen (Ausprägung jeweils 0) ist hinreichend für eine niedrige Extensivität lokaler Klimaschutzmaßnahmen.

Hypothesen $2 a$ und $2 b$ beziehen überlokales Handeln als nahen Faktor in das Erklärungsmodell ein:

H2a Eine hohe Ausprägung der Bedingung überlokalen Handelns ist hinreichend für eine extensive Ausprägung lokaler Klimapolitik. Dies gilt für Fälle, die von nicht eindeutigen Konfigurationen der strukturellen Bedingungen abgedeckt werden. Kontradiktorische Konfigurationen werden durch Einbezug der Bedingungen überlokalen Handelns aufgelöst oder zumindest verringert. Dadurch wird ein höherer Anteil an Fällen erklärt. Die Abdeckung des Gesamtmodells wird durch die Hinzunahme der Bedingungen überlokalen Handelns erhöht.

H2b Eine geringe Ausprägung überlokalen Handelns ist hinreichend für eine geringe Extensivität lokaler Klimaschutzmaßnahmen in Fällen mit nicht eindeutiger Ausprägung struktureller Bedingungen.

Hypothese 3 bezieht sich schließlich auf die Bedingung politischen Unternehmertums:

H3 Aktive politische Unternehmer erklären unter nicht eindeutigen strukturellen Bedingungen und in Abwesenheit überlokaler Aktivitäten eine extensive Klimaschutzpolitik.

\section{Ergebnisse und Diskussion}

Der erste Schritt der QCA ist ein Test notwendiger Bedingungen des untersuchten Outcomes auf Konsistenz. Keine der überprüften Bedingungen erfüllt das Kriterium der Konsistenz. Den höchsten Konsistenzwert besitzt die Bedingung Problembetroffenheit, der mit 0,75 allerdings deutlich unter dem von Schneider und Wagemann (2012, S. 143) vorgeschlagenen ,threshold“ von 0,9 liegt. ${ }^{11}$

Die Analyse hinreichender Bedingungen beginnt mit der Erstellung der Wahrheitstafel ${ }^{12}$, in dieser Untersuchung zunächst für die vier strukturellen bzw. fernen Bedingungen. In dieser Wahrheitstafel sind alle theoretisch möglichen Konfigurationen von Bedingungen aufgelistet, wobei alle 16 Konfigurationen empirisch auftreten. In der nachstehenden Wahrheitstafel (siehe Tab. 3) werden die Konfigurationen, die Anzahl der sie repräsentierenden Fälle und die Konsistenzwerte für den Outcome 1

\footnotetext{
11 Siehe Online-Anhang für die Konsistenzberechnung notwendiger Bedingungen.

12 QCA bildet Fälle als Konfigurationen mehrerer Bedingungen mithilfe von Wahrheitstafeln ab. Fälle mit identischem Outcome können hinsichtlich der Ausprägung dieser Bedingungen überprüft werden. Falls sich zwei Fälle in nur einer Bedingung unterscheiden und in allen anderen gleichen, kann diese Bedingung, weil logisch irrelevant für den Outcome, entfernt werden (Minimierung).
} 
Tab. 3 Wahrheitstafel der strukturellen Faktoren für den Outcome 1 (Kontradiktorische Konfigurationen (mit Konsistenzwerten von $<1$ und $>0$ ) werden in der Outcome-Spalte mit $C$ bezeichnet)

\begin{tabular}{llllllll}
\hline Zeile & B & $P$ & G & W & Fallzahl & OUT & Konsistenz \\
\hline 1 & 1 & 1 & 1 & 1 & 11 & 1 & 1,0 \\
2 & 1 & 0 & 1 & 1 & 2 & 1 & 1,0 \\
3 & 1 & 0 & 1 & 0 & 2 & 1 & 1,0 \\
4 & 0 & 0 & 1 & 0 & 1 & 1 & 1,0 \\
5 & 1 & 1 & 1 & 0 & 5 & $C$ & 0,6 \\
6 & 0 & 1 & 1 & 1 & 4 & $C$ & 0,75 \\
7 & 0 & 1 & 0 & 1 & 3 & $C$ & 0,67 \\
8 & 1 & 1 & 0 & 1 & 5 & $C$ & 0,6 \\
9 & 0 & 1 & 1 & 0 & 3 & $C$ & 0,67 \\
10 & 0 & 0 & 1 & 1 & 5 & $C$ & 0,6 \\
11 & 1 & 1 & 0 & 0 & 5 & $C$ & 0,4 \\
12 & 0 & 1 & 0 & 0 & 10 & $C$ & 0,2 \\
13 & 0 & 0 & 0 & 1 & 5 & $C$ & 0,2 \\
14 & 0 & 0 & 0 & 0 & 6 & 0 & 0,0 \\
15 & 1 & 0 & 0 & 0 & 3 & 0 & 0,0 \\
16 & 1 & 0 & 0 & 1 & 1 & 0 & 0,0 \\
\hline
\end{tabular}

angegeben. ${ }^{13}$ Die Konfigurationen werden nach Konsistenzwerten absteigend geordnet.

Auf Basis dieser Wahrheitstafel können die Hypothesen $1 a$ und $1 b$ überprüft werden. In Zeile 1 sind diejenigen elf Fälle enthalten, in denen alle strukturellen Faktoren den Wert 1 annehmen. Diese Konfiguration ist vollständig konsistent mit der Aussage, dass das Vorhandensein aller strukturellen Faktoren in jedem Fall das Eintreten des Outcomes erklären kann. Gleiches gilt für die Konsistenz der Aussage, dass die Abwesenheit aller strukturellen Faktoren den Nichteintritt des Outcomes erklärt. Der Konsistenzwert von 0 in Zeile 14 bestätigt diese Annahme für die davon abgebildeten sechs Fälle. Damit können die Hypothesen $l a$ und $l b$ angenommen werden.

Allerdings sind diese beiden Konfigurationen nicht die einzigen, die eindeutige Ergebnisse erbringen, die Zeilen 2 bis 4 sowie 15 und 16 enthalten auch widerspruchsfreie Konfigurationen, obwohl nicht alle Bedingungen vorhanden bzw. abwesend sind. Logisch bedeutet dies, dass das Vorhandensein aller Bedingungen zwar hinreichend für das Eintreten des Outcomes ist, dieser aber auch durch andere Konfigurationen erklärt werden kann. Zur umfassenden Analyse aller hinreichenden Konfigurationen wird in einem zweiten Schritt das Minimierungsverfahren ${ }^{14}$ ange-

\footnotetext{
13 Siehe Online-Anhang für den kompletten Datensatz.

14 Alle QCA-Operationen wurden mit den Freeware-Programmen fs/QCA 3.0 und TOSMANA 1.5.2 durchgeführt.
} 
Tab. 4 Hinreichende Konfigurationen struktureller Bedingungen für Outcome 1

\begin{tabular}{llllll}
\hline Zeile & Lösung & Fallzahl & Konsistenz & Rohabdeckung & $\begin{array}{l}\text { Alleinige } \\
\text { Abdeckung }\end{array}$ \\
\hline 1 & $\mathrm{~B}^{*} \mathrm{G}^{*} \mathrm{~W}$ & 13 & 1,0 & 0,35 & 0,35 \\
2 & $\sim \mathrm{W}^{*} \mathrm{G}^{*} \sim \mathrm{P}$ & 3 & 1,0 & 0,08 & 0,08 \\
- & Modellkonsistenz & 1,0 & - & - & - \\
- & Modellabdeckung & 0,43 & - & - & - \\
\hline
\end{tabular}

wendet, das logisch minimale und äquifinale Lösungen ergibt. Die Lösungsformel für den Outcome 1 enthält zwei äquifinale Lösungsterme: ${ }^{15}$

$$
B * G * W+\sim W * G * \sim P \rightarrow O U T
$$

Die Formel enthält zwei hinreichende Konfigurationen struktureller Bedingungen, wobei die Bedingung $G$ (hoher Wählerstimmenanteil der Grünen) in beiden Teilen der Formel enthalten ist. Durch Ausklammern kann die Bedeutung von $G$ unterstrichen werden:

$$
G *(B * W+\sim W * \sim P) \rightarrow O U T
$$

Durch die konservative Anwendung des Verfahrens konnten hinreichende Konfigurationen, die auf jeden Fall zum Eintreten des Outcomes 1 führen, identifiziert werden. Lösungsterm 1 mit 15 Fällen erfüllt dabei die theoretischen Erwartungen (siehe Tab. 4). Wenn die drei Bedingungen hohe Bevölkerungsgröße, hoher Anteil grüner Wählerstimmen und gute wirtschaftliche Situation zutreffen, wird die Problembetroffenheit der Kommune irrelevant. Der zweite Lösungsterm ist weniger intuitiv, da er die Negationen von zwei Bedingungen enthält $(\sim \mathrm{W}, \sim \mathrm{P})$. Quantitativ ist dieser Lösungsterm zwar weniger relevant, da er nur drei empirische Fälle abbildet, er verweist allerdings auf die Bedeutung der Nachfrage nach ökologisch orientierter Politik unter ungünstigen ökonomischen Bedingungen und bei geringer Wahrnehmung des Klimawandels als Problem.

Die ermittelten Konfigurationen decken weniger als die Hälfte aller positiven Fälle ab (Modellabdeckung: 0,43). Alle anderen Fälle mit dem Outcome 1 bleiben unerklärt, da sie in kontradiktorischen Konfigurationen (C) enthalten sind.

Analog zur Analyse hinreichender Bedingungen für das Eintreten des Outcomes werden die hinreichenden Bedingungen des Nichteintritts ermittelt. Wie bereits oben erwähnt, wird Hypothese $1 b$ bestätigt, allerdings finden sich auch widerspruchsfreie

\footnotetext{
15 Die Notation von QCA greift auf boolesche Operatoren zurück. Zum Verständnis dieses Textes genügt die Kenntnis der Operatoren *(UND), + (ODER) und (NICHT). Die boolesche Multiplikation, die dem logischen UND entspricht, wird formal durch das Multiplikationszeichen (*) angezeigt, die boolesche Addition, die dem logischen ODER entspricht, durch das Additionszeichen (+). UND steht für eine Verknüpfung oder Intersektion von Mengen, ODER zeigt eine Vereinigung von Mengen an. Das logische NICHT ( ) zeigt die Negation einer Bedingung an.
} 
Konfigurationen, in denen nicht alle Bedingungen abwesend sind. Die minimierte Lösungsformel für den Outcome 0 lautet:

$$
\sim W * \sim G * \sim P+B * \sim G * \sim P \rightarrow \sim O U T
$$

Der erste Term der Lösungsformel (wirtschaftliche Schwäche, geringer Anteil grüner Wählerstimmen, geringer Problemdruck) beschreibt die theoretisch erwartete Konstellation, dass die konjunktive Abwesenheit der strukturellen Bedingungen geringe Politikinnovation im Klimaschutz erklärt. Der zweite Term verweist darauf, dass die strukturelle Bedingung Bevölkerungsgröße, die für einen großen Verwaltungsapparat steht, alleine nicht in der Lage ist, eine innovative Klimaschutzpolitik zu bewirken. Bei einer schwachen Nachfrage nach ökologischer Politik in Kombination mit geringer Problembetroffenheit fehlt offensichtlich die strukturelle Grundlage zur Erzeugung politischen Drucks zur Beförderung einer extensiven Klimaschutzpolitik.

Die Lösungsformel für den Nichteintritt des Outcomes besitzt eine deutlich geringere Abdeckung $(0,3)$ als die Formel für den Eintritt. ${ }^{16}$ Zusammengenommen bedeutet dies, dass weniger als die Hälfte der Fälle (26 von 71) widerspruchsfrei erklärt werden können. ${ }^{17}$

Dieses Zwischenergebnis rechtfertigt den Einbezug der nahen Faktoren, wobei wir ein mehrteiliges Vorgehen wählen. Zur Überprüfung der Hypothesen $2 a$ und $2 b$ werden die Bedingungen überlokalen Handelns einzeln und in Konjunktion einbezogen. Vertikales überlokales Handeln $(V)$ und horizontales überlokales Handeln $(H)$ gehen zunächst getrennt in das Modell ein. Danach werden beide Bedingungen kombiniert, um die Erklärungskraft des integrierten Konzeptes „überlokales Handeln“ zu prüfen und dem Umstand einer empirisch starken Verflechtung der Dimensionen Rechnung zu tragen. Denn Aktivitäten in beiden Dimensionen gehen oft ineinander über oder bedingen sich gegenseitig (siehe Abschn. 2). Daher bietet es sich an, beide Bedingungen integriert zu betrachten und durch eine UND-Verknüpfung in eine neue Bedingung überlokales Handeln $(\ddot{U} H)$ zu überführen.

Aus Tab. 3 wird ersichtlich, dass lediglich 26 Fälle (16 mit dem Outcome 1 und zehn mit dem Outcome 0) durch strukturelle Bedingungen eindeutig erklärt werden können (Zeilen 1 bis 4 und 14 bis 16). Die restlichen 45 Städte werden durch kontradiktorische Zeilenaussagen abgedeckt. Durch Einbeziehung der Bedingung $V$ können zwei von neun kontradiktorischen Konfigurationen komplett (Tab. 3, Zeilen 8 und 9) und zwei weitere Konfigurationen teilweise theoriekonform aufgelöst werden (Tab. 3, Zeilen 7, 12 und 13). 25 Fälle mit dem Outcome 1 und 23 Fälle mit dem Outcome 0 werden nun erklärt, 23 Fälle verbleiben in kontradiktorischen Konfigurationen.

\footnotetext{
16 Die Asymmetrie der Ergebnisse ist ein Merkmal von QCA. Im Gegensatz zu den gängigen quantitativstatistischen Verfahren geht QCA nicht von symmetrischen Verteilungen, sondern von asymmetrischen Merkmalsbeziehungen aus. Konkret bedeutet dies, dass Bedingungskonfigurationen eines Outcomes nicht automatisch in Negation dessen Ausbleiben erklären können.

17 Aus Gründen der Übersichtlichkeit verzichten wir, abgesehen vom Gesamtmodell, das alle Bedingungen einbezieht, auf eine vollständige Darstellung und Interpretation der Lösungen für das Nichteintreten des Outcomes.
} 
Tab. 5 Hinreichende Bedingungen für Outcome 1 unter Einbezug vertikalen überlokalen Handelns

\begin{tabular}{llllll}
\hline Zeile & Lösung & Fallzahl $^{\mathrm{a}}$ & Konsistenz & Rohabdeckung & $\begin{array}{l}\text { Alleinige } \\
\text { Abdeckung }\end{array}$ \\
\hline 1 & $\mathrm{~B}^{*} \mathrm{~W}^{*} \mathrm{~V}$ & 12 & 1,0 & 0,32 & 0,08 \\
2 & $\sim \mathrm{B}^{*} \mathrm{P}^{*} \mathrm{~W}^{*} \sim \mathrm{V}$ & 3 & 1,0 & 0,08 & 0,08 \\
3 & $\sim \mathrm{B}^{*} \mathrm{G}^{*} \sim \mathrm{W}^{*} \mathrm{~V}$ & 3 & 1,0 & 0,08 & 0,05 \\
4 & $\mathrm{G}^{*} \sim \mathrm{P}^{*} \mathrm{~V}$ & 6 & 1,0 & 0,16 & 0,08 \\
5 & $\mathrm{~B}^{*} \mathrm{G}^{*} \mathrm{~W}$ & 1,0 & 0,35 & 0,11 \\
- & Modellkonsistenz & 1,0 & - & - & - \\
- & Modellabdeckung & 0,68 & - & - & - \\
\hline
\end{tabular}

${ }^{\mathrm{a}}$ Es ist zu beachten, dass die Summe der in der Tabelle enthaltenen Fallzahlen die Gesamtzahl der Fälle mit dem erklärten Outcome 1 überschreitet. Dies hängt damit zusammen, dass ein Fall von mehreren Konfigurationen abgedeckt werden kann und somit auch mehrfach gezählt wird. Für die Gesamtheit der erklärten Fälle ist lediglich die Modellabdeckung $(0,68)$ relevant, die besagt, dass 25 von 37 Fällen mit dem Outcome 1 erklärt werden können

Die Erklärungskraft des Gesamtmodells wird durch den Einbezug vertikalen Handelns deutlich erhöht, die Abdeckung des Modells beträgt 0,68. Tab. 5 präsentiert die Komponenten der sparsamen Lösung. ${ }^{18}$ Die bereits aus dem ersten Analyseschritt bekannte Konfiguration $B * G * W$ behält ihre herausgehobene Erklärungskraft. Durch vertikale Aktivitäten werden allerdings neue Erklärungspfade hinzugefügt. Quantitativ besonders bedeutsam ist Zeile 1, in der hohe vertikale Aktivität in Kombination mit einer hohen Bevölkerungszahl und einer positiven wirtschaftlichen Situation auftritt. Dies verweist auf die Autonomie von Fachverwaltungen größerer Städte mit großen wirtschaftlichen Spielräumen. Die günstige Ressourcenausstattung versetzt sie in die Lage, die Anreizstrukturen vertikaler Aktivitäten im Sinne einer extensiven Adaption von Politikinnovationen zu nutzen. Lösungsterm 4 zeigt zudem, dass eine geringe Problembetroffenheit ,kompensiert“ werden kann durch eine hohe Nachfrage nach grüner Politik in Kombination mit einem hohen vertikalen Aktivitätsniveau. Dies kann als Kombination von „demand-side“- (Nachfrage nach ökologischer Politik) und ,supply-side“-Faktoren interpretiert werden, da überlokales Handeln aktive Verwaltungsakteure voraussetzt, die über genügend Ressourcen verfügen, um sich an Projekten und Ausschreibungen zu beteiligen.

Das Verfahren wird nun angewendet, um eine geringe Diffusion von Politikinnovationen (also den Outcome 0) zu erklären. Die Hinzunahme von $V$ verbessert das Modell noch deutlicher als für die Erklärung eines hohen Niveaus von Politikinnovationen. Die Abdeckung des Modells kann von 0,3 auf 0,68 erhöht werden. Das heißt, dass $68 \%$ der Fälle erklärt bzw. richtig zugeordnet werden. Die Lösung für den Outcome 0 lautet:

$$
\begin{aligned}
& \sim P * \sim G * \sim V+\sim P * \sim G * \sim W+\sim B * \sim W * \sim V \\
& +B * \sim G * W * \sim V+\sim B * P * \sim G * W * V \rightarrow \sim O U T
\end{aligned}
$$

\footnotetext{
18 Zur Ermittlung sparsamer Lösungen werden logical remainders in den Minimierungsprozess einbezogen. Dabei handelt es sich um hypothetische, empirisch aber nicht vorhandene Konfigurationen, mit deren Hilfe übersichtlichere Ergebnisse produziert werden können.
} 
Tab. 6 Hinreichende Bedingungen für Outcome 1 unter Einbezug horizontalen überlokalen Handelns

\begin{tabular}{llllll}
\hline Zeile & Lösung & Fallzahl & Konsistenz & Rohabdeckung & $\begin{array}{l}\text { Alleinige } \\
\text { Abdeckung }\end{array}$ \\
\hline 1 & $\mathrm{G}^{*} \mathrm{H}$ & 10 & 1,0 & 0,27 & 0,05 \\
2 & $\mathrm{~B}^{*} \mathrm{G}^{* \mathrm{~W}}$ & 13 & 1,0 & 0,35 & 0,19 \\
3 & $\mathrm{~W}^{*} \mathrm{P} \mathrm{H}$ & 9 & 1,0 & 0,24 & 0,08 \\
4 & $\sim \mathrm{W}^{*} \mathrm{G}^{*} \mathrm{P}$ & 3 & 1,0 & 0,35 & 0,05 \\
- & Modellkonsistenz & 1,0 & - & - & - \\
- & Modellabdeckung & 0,6 & - & - & - \\
\hline
\end{tabular}

Analog zu vertikalem Handeln wird nun die Bedingung $H$ (horizontales überlokales Handeln) einbezogen. Da das Vorgehen am Beispiel der Bedingung $V$ ausführlich geschildert wurde, kann nun auf eine detaillierte Darstellung verzichtet werden. Auch durch Einbeziehung von $H$ können zwei widersprüchliche Konfigurationen aufgelöst werden. Darunter ist Zeile 5 aus Tab. 3, die durch die Hinzunahme von $V$ nicht aufgelöst werden konnte. Durch die Hinzunahme von $H$ wird die Abdeckung des Modells ebenfalls deutlich erhöht, sie beträgt 0,6. Die Lösung enthält vier Konfigurationen (siehe Tab. 6), wovon drei quantitativ besonders bedeutsam sind. Die Zeilen 2 und 4 in Tab. 6 sind bereits aus dem Modell der strukturellen Bedingungen bekannt und benötigen keine weitere Erläuterung. Somit wird deutlich, in welchen Kontexten horizontales überlokales Handeln eine eigenständige Erklärungskraft besitzt. Es ist entweder in Kombination mit einer starken Nachfrage nach grüner Politik (Tab. 6, Zeile 1) oder in Kombination mit wirtschaftlicher Stärke und hoher Problembetroffenheit (Tab. 6, Zeile 3) hinreichend zur Erklärung der Übernahme von Politikinnovationen. Damit wird deutlich, dass überlokale Aktivitäten in der horizontalen Dimension eine verstärkende Wirkung in Kontexten besitzen, die bereits vorteilhaft für ein hohes Ausmaß an Politikinnovationen sind.

Das Ausbleiben von Politikinnovationen kann hingegen weniger gut mit dem Einbezug horizontalen Handelns erklärt werden. Die Abdeckung der Lösung erhöht sich lediglich auf 0,38. Damit ist es nicht möglich, eine sinnvolle Aussage über die Wirkung geringen überlokalen Handelns in horizontaler Dimension zu treffen.

Im Sinne des oben eingeführten einheitlichen Konzeptes überlokalen Handelns werden im nächsten Schritt beide Bedingungen $V$ und $H$ gemeinsam einbezogen. Um eine Proliferation hypothetischer Konfigurationen zu vermeiden, werden beide Bedingungen durch eine UND-Verknüpfung in der neuen Bedingung überlokales Handeln $(\ddot{U} H)$ integriert. Da es sich dabei um ein strenges Kriterium handelt (V und $H$ müssen mit 1 kodiert sein), ist mit einer relativ geringen Modellabdeckung zu rechnen bei gleichermaßen sparsameren Lösungen. Die Zusammenfassung (siehe Tab. 7) zeigt eine Abdeckung von 0,6, d.h. 22 der Fälle mit einem Outcome von 1 werden durch die Lösungsformeln abgedeckt. Daneben erhält die Lösung eine relativ große Trennschärfe, da nur sechs Fälle von mehr als einer Konfiguration abgedeckt werden. Als größter Vorzug des integrierten Ansatzes erscheint, dass überlokales Handeln als hinreichende Bedingung zur Erklärung für den Outcome 1 gelten kann (Tab. 7, Zeile 1). Alle 13 Städte, die ein hohes Maß an überlokalen 
Tab. 7 Hinreichende Bedingungen für Outcome 1 unter Einbezug überlokalen Handelns

\begin{tabular}{llllll}
\hline Zeile & Lösung & Fallzahl & Konsistenz & Rohabdeckung & $\begin{array}{l}\text { Alleinige } \\
\text { Abdeckung }\end{array}$ \\
\hline 1 & $\ddot{U} \mathrm{H}$ & 13 & 1,0 & 0,35 & 0,16 \\
2 & $\sim \mathrm{W}^{*} \mathrm{G}^{*} \sim \mathrm{P}$ & 3 & 1,0 & 0,08 & 0,05 \\
3 & $\mathrm{~B}^{*} \mathrm{G}^{* \mathrm{~W}}$ & 13 & 1,0 & 0,35 & 0,19 \\
- & Modellkonsistenz & 1,0 & - & - & - \\
- & Modellabdeckung & 0,6 & - & - & - \\
\hline
\end{tabular}

Aktivitäten in beiden Dimensionen aufweisen, weisen eine hohe Übernahme von Politikinnovationen auf.

Die drei Lösungsterme erlauben eine Reihe von Schlussfolgerungen. Lösungsterm 1 beschreibt im überlokalen Handlungsraum aktive Städte. Die Kombination beider Formen überlokalen Handelns erweist sich als hinreichend für ein hohes Innovationsniveau. $H 2 a$ kann somit bestätigt werden. Auch wenn keine validen Aussagen über Wirkmechanismen getroffen werden können, schlagen sich die unterstellten Wirkungen überlokalen Handelns, wie Lernen oder die Verpflichtung auf bestimmte Ziele, offenbar tatsächlich in Maßnahmen nieder. Die weiteren zwei Lösungsterme sind bereits bekannt aus der Analyse der strukturellen Faktoren. Überlokales Handeln löst eine Reihe von kontradiktorischen Konfigurationen auf, die Anzahl unerklärter Fälle wird verringert. Überlokales Handeln erhöht dabei nicht nur die Abdeckung des Modells, sondern stellt einen alternativen Erklärungspfad zur Verfügung, der die strukturellen Erklärungspfade ergänzt. Dies deutet darauf hin, dass überlokales Handeln durchaus eine kompensatorische Funktion übernehmen kann. Allerdings kann angesichts des querschnittsorientierten Untersuchungsdesigns die Frage nicht beantwortet werden, wie stark der Effekt überlokalen Handelns unabhängig von den gegebenen strukturellen Voraussetzungen tatsächlich einzuschätzen ist. Aufgrund des strengen Verknüpfungskriteriums, das zudem zu einer gewissen „Windschiefe" der Verteilung bei der Bedingung $\ddot{U} H$ führt (Schneider u. Wagemann, S. $232 \mathrm{ff}$.) ${ }^{1920}$, bleiben 15 der 37 Fälle mit dem Outcome 1 letztlich unerklärt.

Das Ergebnis der Analyse der hinreichenden Bedingungen für das Nichteintreten des Outcomes erlaubt bei Anwendung des strengen Konsistenzkriteriums ähnlich wie die Analyse horizontalen Handelns keine belastbaren Aussagen über dessen

\footnotetext{
19 Windschiefe (,skewedness“) besagt, dass die Kalibrierung zu einer gewissen Ungleichverteilung führt. Einer geringen (hohen) Zahl von Bedingungen mit dem Wert 0 steht eine hohe (geringe) Zahl von Bedingungen mit dem Wert 1 gegenüber. Problematisch ist insbesondere der Effekt von ,simultaneous subset relations", d. h. dass sich Mengen stark überschneiden (Schneider und Wagemann 2012, S. 238). In unserer Analyse kommt dies dadurch zum Ausdruck, dass eine starke Überlagerung zwischen der Konfiguration, die alleine durch überlokales Handeln beschrieben wird (siehe Tab. 7 und 8), und denjenigen Konfigurationen, die durch strukturelle Bedingungen gebildet werden, besteht. Um relativ sparsame Lösungen zu erhalten, die zudem angeben, unter welchen Bedingungen auf jeden Fall innovative Maßnahmen übernommen werden (siehe Abschn. 3.2), erscheint die Anwendung des strengen Verknüpfungskriteriums gerechtfertigt. Trotz der Überlagerung spricht für das Verfahren zudem die Möglichkeit äquifinale Lösungspfade klar herauszustellen.

${ }^{20}$ Für die „Windschiefe“ ist auch die „Ungleichverteilung“ bei Bedingung $H$ verantwortlich, die allerdings durch die theoretische Begründung der Kalibrierung (siehe Abschn. 3.3.3) gerechtfertigt wird.
} 
mögliche Wirkungen. Daher haben wir uns entschieden, in diesem Fall von unserem konservativen Verfahren abzuweichen und auch solche Konfigurationen mit $1 \mathrm{zu}$ kodieren, die nicht vollständig konsistent mit der Aussage sind, dass der Outcome eintritt. Betroffen sind davon zwei Konfigurationen, die dennoch einen relativ hohen Konsistenzwert von 0,8 besitzen. Zu beachten ist allerdings, dass die Lösungsformel nun Terme enthält, in denen der Outcome nicht in jedem Fall eintritt, sondern nur in einer sehr großen Anzahl von Fällen. Die Abdeckung der Lösung beträgt 0,7 bei einer Gesamtkonsistenz von 0,89:

$$
\sim P * \sim G+\sim B * \sim G * \sim W+B * \sim G * W * \sim \ddot{U} H \rightarrow \sim O U T
$$

Während die ersten beiden Lösungsterme lediglich strukturelle Bedingungen (in Abwesenheit) enthalten, enthält der dritte Term überlokales Handeln als INUS-Bedingung. Es sollte beachtet werden, dass dieser Term in identischer Struktur bereits in dem Modell enthalten ist, welches alleine die vertikalen Aktivitäten berücksichtigt. Eine geringe Nachfrage nach ökologischer Politik und eine geringe Bereitschaft von Verwaltung und Politik, den überlokalen Handlungsraum zur Etablierung und Durchsetzung extensiver Klimaschutzmaßnahmen zu nutzen, erklären ein niedriges Niveau der Adaption innovativer Maßnahmen.

Der Einbezug überlokalen Handelns als integrierte Bedingung erhöht die Erklärungskraft des Modells deutlich, allerdings bleiben 15 Fälle mit dem Outcome 1 unerklärt. Hypothese $2 b$ wird nicht umfassend bestätigt, da ein niedriges Niveau überlokalen Handelns lediglich in einem Teil der Lösungsformel auftaucht. Im Hinblick auf die Erklärung geringer Maßnahmenextensivität erhält das Modell nur dann eine gute Erklärungskraft, wenn das Konsistenzkriterium herabgesetzt wird.

Im letzten Schritt der Untersuchung erfolgt daher die Einbeziehung der Bedingung OberbürgermeisterIn als aktiver politischer Unternehmer $(U)$. Hypothese 3 postuliert, dass ,,policy entrepreneurship“ ungünstige Rahmenbedingungen substituiert. Dahinter steht die theoretische Annahme, dass eine engagierte politische Führung ähnlich wirken kann wie überlokale Aktivitäten. Dies könnte insbesondere in kleineren Städten, die lediglich über begrenzte Ressourcen zur Beteiligung an überlokalen Aktivitäten verfügen, relevant werden. Technisch bedeutet der postulierte Effekt die Auflösung weiterer kontradiktorische Konfigurationen und eine weitere Erhöhung der Modellabdeckung.

Der Einbezug der Bedingung $U$ trägt zur Auflösung weiterer Widersprüche und zu einer höheren Abdeckung des Gesamtmodells bei. Nun werden 28 von 37 Fällen mit dem Outcome 1 erklärt, neun Fälle verbleiben in kontradiktorischen Konfigurationen. $\mathrm{Zu}$ bedenken ist allerdings, dass bei einer QCA mit sechs Bedingungen zwangsläufig zwei Probleme auftauchen, zum einen eine Proliferation von logical remainders, zum anderen eine Individualisierung von Fällen. Dies zeigt sich daran, dass von den hypothetisch möglichen 64 Konfigurationen lediglich 32 empirisch vorhanden sind und 16 Konfigurationen nur einen Fall abdecken.

Die sparsame Lösung weist fünf Lösungsterme auf, die drei unterschiedliche Gruppen abbilden (siehe Tab. 8). Die ersten beiden Gruppen sind aus der bisherigen Analyse bekannt. Sie werden entweder durch eine hohe überlokale Aktivität oder durch strukturelle Bedingungen abgebildet (Tab. 8, Zeilen 1-3). Hinzugekommen 
Tab. 8 Hinreichende Bedingungen für Outcome 1 unter Einbezug überlokalen Handelns und politischen Unternehmertums

\begin{tabular}{llllll}
\hline Zeile & Lösung & Fallzahl & Konsistenz & Rohabdeckung & $\begin{array}{l}\text { Alleinige } \\
\text { Abdeckung }\end{array}$ \\
\hline 1 & $\ddot{\mathrm{U} H}$ & 13 & 1,0 & 0,35 & 0,08 \\
2 & $\mathrm{~B}^{*} \mathrm{G}^{*} \mathrm{~W}$ & 13 & 1,0 & 0,35 & 0,16 \\
3 & $\sim \mathrm{P}^{*} \mathrm{G}^{*} \sim \mathrm{W}$ & 3 & 1,0 & 0,08 & 0,05 \\
4 & $\mathrm{G}^{*} \mathrm{U}$ & 12 & 1,0 & 0,32 & 0,08 \\
5 & $\mathrm{P}^{*} \mathrm{~W} * \mathrm{U}$ & 6 & 1,0 & 0,16 & 0,03 \\
- & Modellkonsistenz & 1,0 & - & - & - \\
- & Modellabdeckung & 0,76 & - & - & - \\
\hline
\end{tabular}

Tab. 9 Hinreichende Bedingungen für Outcome 0 unter Einbezug überlokalen Handelns und politischen Unternehmertums

\begin{tabular}{llllll}
\hline Zeile & Lösung & Fallzahl & Konsistenz & Rohabdeckung & $\begin{array}{l}\text { Alleinige } \\
\text { Abdeckung }\end{array}$ \\
\hline 1 & $\sim \mathrm{P}^{*} \sim \mathrm{G}^{*} \sim \mathrm{W}$ & 9 & 1,0 & 0,26 & 0,09 \\
2 & $\mathrm{~B}^{*} \sim \mathrm{G}^{*} \mathrm{~W} \sim \mathrm{U} \mathrm{H}$ & 3 & 1,0 & 0,09 & 0,06 \\
3 & $\sim \mathrm{P}^{*} \sim \mathrm{G}^{*} \mathrm{U}$ & 2 & 1,0 & 0,08 & 0,03 \\
4 & $\sim \mathrm{B}^{*} \mathrm{P}^{*} \mathrm{G}^{*} \sim \mathrm{U}$ & 2 & 1,0 & 0,06 & 0,06 \\
5 & $\sim \mathrm{B}^{*} \sim \mathrm{G}^{*} \sim \mathrm{W}^{*} \sim \mathrm{U}$ & 13 & 0,93 & 0,38 & 0,21 \\
- & Modellkonsistenz & 0,96 & - & - & - \\
- & Modellabdeckung & 0,65 & - & - & - \\
\hline
\end{tabular}

sind zwei Lösungsterme, in denen aktives politisches Unternehmertum als INUSBedingung auftaucht. Diese können als dritte Gruppe interpretiert werden. Zeile 4 in Tab. 8 beschreibt Städte, die starke ökologische Parteien und einen aktiven politischen Unternehmer im Amt des Oberbürgermeisters aufweisen. Hier greift eine präferenzorientierte Erklärung, die auf eine Übereinstimmung von politischer Nachfrage und politischem Angebot abhebt. Empirisch umfasst diese hinreichende Konfiguration eine Reihe kleinerer Großstädte, die begrenzte administrative Kapazitäten und teilweise geringe überlokale Aktivitäten aufweisen. Dies unterstützt Hypothese 3 und den darin formulierten Kompensationseffekt. Der Lösungsterm in Tab. 8, Zeile 5 verweist auf Städte mit einer hohen Problembetroffenheit in Kombination mit einer positiven wirtschaftlichen Situation. Unter diesen bereits günstigen strukturellen Rahmenbedingungen kann ein aktiver politischer Unternehmer offensichtlich den Ausschlag zu einer extensiven Klimaschutzpolitik geben.

Während das Modell ein hohes Maß an Diffusion innovativer Policies recht umfassend erklären kann, ist die Erklärungskraft für eine geringe Übernahme begrenzter. Auch durch Hinzunahme der Bedingung $U$ bleibt die Abdeckung bei einem Konsistenzmaß von 1 auf einem niedrigen Wert von 0,53, d.h. 18 der 34 Fälle mit dem Outcome 0 werden erklärt. Diese verteilen sich zudem auf sechs Lösungsterme. Wenn das Konsistenzkriterium ermäßigt wird (Einbezug einer Konfiguration mit einem Konsistenzwert von 0,93), ergeben sich gleichermaßen eine höhere Abdeckung und eine weitere Minimierung der Lösungsterme. Bei einer Abdeckung von 0,65 werden 22 von 34 Fällen mit dem Outcome 0 erklärt (siehe Tab. 9). 
Die Zeilen 1 und 2 in Tab. 9 beschreiben bereits bekannte Konfigurationen. Die Bedingung überlokales Handeln $(\ddot{U} H)$ taucht in der zweiten Lösung als abwesend auf, was den Erwartungen entspricht. Die Bedingung aktiver politischer Unternehmer $(U)$ erscheint allerdings in beiden möglichen Ausprägungen in den Lösungen. Lösungsterm 3 beschreibt Städte, in denen ein niedriges Niveau an Politikinnovationen trotz eines aktiven Oberbürgermeisters besteht. In Kombination mit einer geringen Problemwahrnehmung und einer geringen politischen Nachfrage bleibt der Spielraum eines aktiven politischen Unternehmers offensichtlich eingeschränkt. Zeile 4 beschreibt empirisch eine Konstellation von kleinen Städten, in denen eine geringe Extensivität von Klimaschutzmaßnahmen bei hoher Problembetroffenheit und hoher politischer Nachfrage besteht. Dieser auf den ersten Blick nicht intuitive Befund wird etwas plausibler, wenn die Bedingung $\sim \mathrm{U}$ beachtet wird, Klimaschutzbelange also keine herausgehobene politische Unterstützung erhalten. $\mathrm{Zu}$ beachten ist allerdings, dass die Lösungsterme 3 und 4 lediglich eine geringe Anzahl an Fällen abdecken. Quantitativ bedeutsamer ist der fünfte Lösungsterm, der allerdings nicht vollständig konsistent ist. Da alle Bedingungen in Negation auftreten (also nicht vorhanden sind), entspricht er den theoretischen Erwartungen. Interessant ist hier vielmehr der eine Fall, der den Outcome 1 besitzt, was zu einer Konsistenz von 0,96 führt. $^{21}$

\section{Fazit}

Ausgehend von einem breiten Verständnis von Klimaschutz wurde in diesem Artikel ein Erklärungsmodell für die Adaption innovativer Klimaschutzmaßnahmen in deutschen Großstädten entwickelt. Auf Basis einer Literaturrecherche und theoretischer Überlegungen zu Determinanten der Staatstätigkeit wurden zunächst „ferne“ (strukturelle) Bedingungen eines solchen Modells identifiziert und operationalisiert. Dazu zählen Bevölkerungsgröße, eine günstige wirtschaftliche Situation, hoher Wähleranteil der Grünen oder anderer ökologischer Listen sowie eine hohe Problembetroffenheit. Diese Bedingungen liefern bereits eine hinreichende Erklärung für die Extensivität von beschlossenen Maßnahmen, decken jedoch nur den kleineren Teil der untersuchten Städte ab.

Die zentrale Fragestellung des Artikels zielte auf den Beitrag überlokaler Aktivitäten zum Umfang (Extensivität) lokaler Klimaschutzmaßnahmen. Diese wurden in einem zweistufigen Analyseprozess in das Modell integriert. Ebenso wurde besonderes politisches Engagement durch die OberbürgermeisterInnen (als politische Unternehmer) einbezogen. Beide Aspekte sind im Gegensatz zu den strukturellen Kontextfaktoren näher am Outcome und leichter durch Akteure zu beeinflussen. Sie werden daher als ,nahe“ Faktoren bezeichnet.

\footnotetext{
21 Dieser Befund kann zur Auswahl eines relevanten Falls für Fallstudien genutzt werden, die in der zweiten Phase des Forschungsprojektes (siehe Fußnote 1) durchgeführt werden.
} 
Die Untersuchung unter Einbezug der nahen Faktoren überlokalen Handelns und politischer Unternehmer führt zu mehreren äquifinalen Erklärungspfaden. Diese können in drei Gruppen eingeteilt werden, wodurch unterschiedliche Bedingungen hervorgehoben werden:

1. In der ersten Gruppe bieten strukturelle Bedingungen eine hinreichende Erklärung für ein hohes Maß an Politikinnovationen. Eine herausgehobene Bedeutung kommt dabei der elektoralen Stärke grüner/ökologischer Parteien zu, die in beiden Lösungspfaden präsent ist. Damit werden traditionelle Annahmen der Staatstätigkeitsforschung bestätigt, allerdings unter der Einschränkung einer relativ geringen Anzahl erklärter Fälle.

2. In der zweiten Gruppe bietet ein hohes Niveau an überlokalen Aktivitäten als integrierte Bedingung aus vertikalem und horizontalem Handeln ebenso eine hinreichende Erklärung. Dies unterstützt die theoretische Annahme, dass die Einbindung in überlokale Zusammenhänge und die strategische Nutzung des überlokalen Handlungsraums durch lokale Akteure einen identifizierbaren Beitrag zur Diffusion innovativer Politik leisten. Werden die Dimensionen überlokalen Handelns getrennt betrachtet, besitzt vertikales Handeln eine höhere Erklärungskraft. Im methodischen Kontext von QCA heißt das, dass die „Abdeckung“ des Modells erhöht wird. Horizontales Handeln ist hingegen besonders in Städten ausgeprägt, die aufgrund der strukturellen Bedingungen eine hohe Maßnahmenextensivität aufweisen. Ihm kommt somit eher eine verstärkende oder unterstützende Funktion in Städten mit bereits vorteilhaften Kontextbedingungen zu. Durch die Analyse konnten vorliegende Forschungsergebnisse (Hakelberg 2014, Sharp et al. 2011) über die Wirkung überlokalen Handelns bestätigt und kontextualisiert werden, d.h. es konnte gezeigt werden, unter welchen Bedingungen (hohe gesellschaftliche Nachfrage nach Klimaschutz oder eine hohe städtische Betroffenheit durch den Klimawandel) überlokales Handeln wirksam wird.

3. In der dritten Gruppe wird die Rolle politischer Unternehmer deutlich. Die Analyse bestätigt auch die Befunde, die Krause $(2011,2013)$ für amerikanische Städte erarbeitet hat. Sie gehen aber auch darüber hinaus, in dem durch die konfigurative Herangehensweise spezifische Kontexte identifiziert werden, in denen politische Unternehmer einen Unterschied machen. Die besondere Förderung von Klimaschutz durch die politische Führung kann in Fällen, die durch nicht eindeutige Rahmenbedingungen sowie geringe überlokale Aktivitäten geprägt sind, eine kompensatorische Funktion entfalten.

Insgesamt wird die Erklärungskraft des Modells durch die Integration der nahen Faktoren verbessert. Eine Reihe von Widersprüchen innerhalb des Modells kann aufgelöst werden, die Lösungspfade erhalten eine größere Trennschärfe. Zudem wird die Abdeckung des Erklärungsmodells deutlich erhöht: durch die Einbeziehung überlokalen Handelns und aktiver OberbürgermeisterInnen als politische Unternehmer werden 28 von 37 Fällen mit dem Outcome 1 erklärt. Die Berücksichtigung überlokalen Handelns und politischer Unternehmer zeigt, dass günstige Kontextbedingungen verstärkt und ungünstige Kontextbedingungen zum Teil kompensiert werden können. 
Einschränkend ist anzumerken, dass das Modell das Ausbleiben von Innovationen bzw. eine geringe Extensivität lokale Klimaschutzpolitik weniger gut erklären kann: 22 von 34 nicht-innovativen Städten (Outcome 0) werden durch das Modell abgedeckt. Diese Asymmetrie spricht einerseits für die Anwendung der Methode, da QCA als asymmetrisches Verfahren in der Lage ist, genau solche Phänomene abzubilden. Andererseits werden aber auch Grenzen des Erklärungsmodells aufgezeigt, die zum einen auf nicht berücksichtigte Faktoren, zum anderen aber auch auf idiosynkratrische (Scharpf 2000, S. 84) stadtinterne Prozesse zurückgehen können. Dies umfasst auch die Frage nach einem adäquaten „Framing“, das stadtspezifische Gegebenheiten strategisch berücksichtigt (Heinelt und Lamping 2015).

Ebenfalls unberücksichtigt blieb in dieser Untersuchung der temporale bzw. prozessorale Aspekt. Aufgrund des Untersuchungsdesigns war es nicht möglich, die Entwicklung politisch-administrativer Prozesse, überlokaler Aktivitäten und das Engagement politischer Unternehmer im Längsschnitt zu analysieren. Abschließende Aussagen über den Einfluss überlokalen Handelns werden dadurch natürlich beschränkt. Die Analyseergebnisse zu überlokalem Handeln verweisen aber auf drei Aspekte: Erstens existiert zwar eine gewisse Übereinstimmung von Städten mit günstigen strukturellen Rahmenbedingungen und hohen überlokalen Aktivitäten. In allen drei Analysevarianten, in denen überlokales Handeln berücksichtigt wird (vertikal, horizontal oder kombiniert), verbessert sich die Erklärungskraft des Modells jedoch deutlich. Dies bedeutet, dass eine hohe überlokale Aktivität unter nicht eindeutigen strukturellen Bedingungen den Ausschlag zu einer extensiven Klimaschutzpolitik gibt. Dies hängt, zweitens, damit zusammen, dass überlokales Handeln zum einen eine größere Vielfalt an Handlungsoptionen eröffnet, zum anderen aber auch eine selbstverpflichtende Wirkung entfaltet. Schließlich signalisiert überlokales Handeln, drittens, eine Offenheit gegenüber Innovationen und damit die Bereitschaft neue Maßnahmen schneller zu adaptieren.

Die QCA ermöglichte eine Bestätigung und Kontextualisierung der theoretisch angenommenen Wirkung überlokalen Handelns. Für eine genauere Prüfung des Zusammenhangs sind jedoch vertiefende Fallstudien notwendig. Hier zeigt sich eine weitere Stärke der fallorientierten Methode, die eine Grundlage für die Auswahl von Fallstudien bietet. Vor allem die widersprüchlichen Konfigurationen, die durch die Analyse nicht aufgelöst werden konnten, bieten einen guten Ansatz zur Fallauswahl für weitergehende Untersuchungen.

Open Access Dieser Artikel wird unter der Creative Commons Namensnennung 4.0 International Lizenz (http://creativecommons.org/licenses/by/4.0/deed.de) veröffentlicht, welche die Nutzung, Vervielfältigung, Bearbeitung, Verbreitung und Wiedergabe in jeglichem Medium und Format erlaubt, sofern Sie den/die ursprünglichen Autor(en) und die Quelle ordnungsgemäß nennen, einen Link zur Creative Commons Lizenz beifügen und angeben, ob Änderungen vorgenommen wurden.

\section{Literatur}

Barber, Benjamin. 2013. If mayors ruled the world. Dysfunctional nations, rising cities. New Haven, London: Yale University Press.

Bendlin, Lena. 2016. Cities' views and ownership of the covenant of mayors. In Städte und Energiepolitik im europäischen Mehrebenensystem. Zwischen Energiesicherheit, Nachhaltigkeit und Wettbewerb, Hrsg. Jörg Kemmerzell, Michèle Knodt, und Anne Tews, 103-124. Baden-Baden: Nomos. 
Berg-Schlosser, Dirk, und Lasse Cronqvist. 2012. Aktuelle Methoden der Vergleichenden Politikwissenschaft. Einführung in konfigurationelle (QCA) und makro-quantitative Verfahren. Opladen, Farmington Hills: Barbara Budrich.

Bulkeley, Harriet, und Michele Betsill. 2013. Revisiting the urban politics of climate change. Environmental Politics 22(1):136-154. https://doi.org/10.1080/09644016.2013.755797.

Bulkeley, Harriet, und Kristine Kern. 2006. Local government and the governing of climate change in Germany and the UK. Urban Studies 43(12):2237-2259. https://doi.org/10.1080/00420980600936491.

Bulkeley, Harriet, Liliana Andonova, Karin Bäckstrand, Michele Betsill, Daniel Compagnon, Rosaleen Duffy, Ans Kolk, Matthew Hoffmann, David Levy, Peter Newell, Tori Milledge, Matthew Paterson, Philipp Pattberg, und Stacy VanDeveer. 2012. Governing climate change transnationally: assessing the evidence from a database of sixty initiatives. Environment and planning C: Government and Policy 30(4):591-612. https://doi.org/10.1068/c11126.

Busch, Henner. 2015. Linked for action? An analysis of transnational municipal climate networks in Germany. International Journal of Urban Sustainable Development 7(2):213-231. https://doi.org/10. 1080/19463138.2015.1057144.

Difu - Deutsches Institut für Urbanistik. 2013. Kommunaler Klimaschutz, erneuerbare Energien und Klimawandel in Kommunen. http://edoc.difu.de/edoc.php?id=AL7HYEJX. Zugegriffen: 10. Mai 2018.

Emmenegger, Patrick. 2011. Job security regulations in western democracies. A fuzzy set analysis. European Journal of Political Research 50(3):336-364. https://doi.org/10.1111/j.1475-6765.2010.01933. $\mathrm{x}$.

Gissendanner, Scott. 2002. Die Bedeutung des Bürgermeisters für die strategische Entscheidungsfähigkeit deutscher Großstädte. In Kommunale Entscheidungsprozesse im Wandel. Theoretische und empirische Analysen, Hrsg. Jörg Bogumil, 91-109. Wiesbaden: Springer.

Goertz, Gary, und James Mahoney. 2012. A tale of two cultures. Qualitative and quantitative research in the social sciences. Princeton: Princeton University Press.

Hakelberg, Lukas. 2014. Governance by diffusion. Transnational municipal networks and the spread of local climate strategies in europe. Global Environmental Change 14(1):107-129. https://doi.org/10. 1162/GLEP_a_00216.

Heinelt, Hubert, und Wolfram Lamping. 2015. Wissen und Entscheiden. Lokale Strategien gegen den Klimawandel in Frankfurt am Main, München und Stuttgart. Frankfurt am Main: Campus.

Holzinger, Katharina, Helge Jörgens, und Christoph Knill (Hrsg.). 2007. Transfer, Diffusion und Konvergenz von Politiken. Wiesbaden: Springer VS.

Homsy, George C., und Mildred E. Warner. 2015. Cities and Sustainability. Polycentric action and multilevel governance. Urban Affairs Review 51(1):46-73. https://doi.org/10.1177/1078087414530545.

Hörisch, Felix. 2013. Fiscal policy in hard times. A fuzzy-set QCA of fiscal policy reactions to the financial crisis. Zeitschrift für vergleichende Politikwissenschaft 7(2):117-141. https://doi.org/10.1007/ s12286-013-0152-y.

Jordan, Andrew, und Dave Huitema. 2014. Innovations in climate policy: the politics of invention, diffusion, and evaluation. Environmental Politics 23(5):715-734. https://doi.org/10.1080/09644016.2014. 923614.

Kemmerzell, Jörg, und Anne Tews. 2014. Akteursorientierungen im überlokalen Handlungsraum. Herausforderungen und Chancen lokaler Klimapolitik im Mehrebenensystem. dms - der moderne staat - Zeitschrift für Public Policy, Recht und Management 7(2):269-287. https://doi.org/10.3224/dms. v7i2.17317.

Kern, Kristine. 2014. Climate governance in the European Union multilevel system: The role of cities. In Multilevel environmental governance. Managing water and climate change in europe and north america, Hrsg. Inger Weibust, James Meadowcroft, 111-130. Cheltenham, Northampton: Edward Elgar.

Kern, Kristine, und Harriet Bulkeley. 2009. Cities, Europeanization and multi-level governance: Governing climate change through transnational municipal networks. Journal of Common Market Studies 47(2):309-332. https://doi.org/10.1111/j.1468-5965.2009.00806.x.

Kern, Kristine, Stefan Niederhafner, Sandra Rechlin, und Jost Wagner. 2005. Kommunaler Klimaschutz in Deutschland. Handlungsoptionen, Entwicklung und Perspektiven. WZB Discussion Paper SP IV 2005-101. https://www.ssoar.info/ssoar/bitstream/handle/document/19672/ssoar-2005-kern_et_alkommunaler_klimaschutz_in_deutschland_-.pdf?sequence=1. Zugegriffen: 20. April 2018.

Knill, Christoph, Marc Debus, und Stephan Heichel. 2010. Do parties matter in Internationalised policy areas? European Journal of Political Research 49(3):301-336. https://doi.org/10.1111/j.1475-6765. 2009.01903.x. 
Knill, Christoph, Kai Schulze, und Jale Tosun. 2012. Regulatory policy outputs and impacts: Exploring a complex relationship. Regulation \& Governance 6(4):427-444. https://doi.org/10.1111/j.17485991.2012.01150.x.

Knodt, Michèle. 2010. Kommunales Regieren im europäischen Mehrebenensystem. In Die EU-Reflexionsgruppe ,Horizont 2020-2030': Herausforderungen und Reformoptionen für das Mehrebenensystem, Hrsg. Gabriele Abels, Annegret Eppler, und Michèle Knodt, 153-168. Baden-Baden: Nomos.

Knodt, Michèle, und Martin Große Hüttmann. 2012. Der Multi-Level Governance-Ansatz. In Theorien der europäischen Integration, Hrsg. Hans-Jürgen Bieling, Marika Lerch, 187-205. Wiesbaden: Springer VS.

Krause, Rachel M. 2011. Symbolic or substantive policy? Measuring the extent of local commitment to climate protection. Environment and Planning C: Government and Policy 29(1):46-62. https://doi. org/10.1068/c09185.

Krause, Rachel M. 2013. The motivations behind municipal climate engagement. Cityscape 15(1):125-141.

Lee, Taedong, und Chris Koski. 2015. Multilevel governance and urban climate change mitigation. Environment and Planning C: Government and Policy 33(6):1501-1517. https://doi.org/10.1177/ $0263774 X 15614700$.

Legewie, Nicolas. 2013. An introduction to applied data analysis with qualitative comparative analysis (QCA). Forum: Qualitative Social Research 14(3). https://doi.org/10.17169/fqs-14.3.1961.

Lijphart, Arend. 1975. The comparable cases strategy in comparative research. Comparative Political Studies 8(2):158-177.

Mayer, Ines, Volker Schneider, und Claudius Wagemann. 2011. Energieeffizienz in privaten Haushalten im internationalen Vergleich. Eine Policy-Wirkungsanalyse mit QCA. Politische Vierteljahresschrift 52(3):399-423.

Mintrom, Michael, und Joannah Luetjens. 2017. Policy entrepreneurs and problem framing. The case of climate change. Environment and Planning C: Politics and Space 35(8):1362-1377. https://doi.org/ $10.1177 / 2399654417708440$.

Pitt, Damian. 2010. The impact of internal and external characteristics on the adoption of climate mitigation policies by US municipalities. Environment and Planning C: Government and Policy 28(5):851-871. https://doi.org/10.1068/c09175.

Ragin, Charles. 1987. The comparative method. Moving beyond qualitative and quantitative strategies. Berkeley: University of California Press.

Ryan, Daniel. 2015. From commitment to action. A literature review on climate policy implementation at city level. Climatic Change 131(4):519-529. https://doi.org/10.1007/s10584-015-1402-6.

Schaffrin, André, Sebastian Sewerin, und Sibylle Seubert. 2015. Toward a comparative measure of climate policy output. Policy Studies Journal 43(2):257-282. https://doi.org/10.1111/psj.12095.

Scharpf, Fritz W. 2000. Interaktionsformen. Akteurszentrierter Institutionalismus in der Politikforschung. Opladen: Leske + Budrich.

Schmidt, Manfred G. 1996. When parties matter: A review of the possibilities and limits of partisan influence on public policy. European Journal of Political Research 30(2):155-183. https://doi.org/10. 1111/j.1475-6765.1996.tb00673.x.

Schneider, Carsten Q., und Claudius Wagemann. 2012. Set-theoretic methods for the social sciences. A guide to qualitative comparative analysis. Cambridge: Cambridge University Press.

Sharp, Elaine B., Dorothy M. Daley, und Michael S. Lynch. 2011. Understanding local adoption and implementation of climate change mitigation policy. Urban Affairs Review 47(3):433-457. https://doi. org/10.1177/1078087410392348.

Siewert, Markus B. 2017. Qualitative comparative analysis. In Neue Trends in den Sozialwissenschaften. Innovative Techniken für qualitative und quantitative Forschung, Hrsg. Sebastian Jäckle, 273-305. Wiesbaden: Springer VS.

Stoiber, Michael, und Annette Elisabeth Töller. 2016. Ursachen der Privatisierung des Maßregelvollzugs in Deutschland. Eine QCA im Bundesländervergleich. Zeitschrift für Vergleichende Politikwissenschaft 10(1):9-36. https://doi.org/10.1007/s12286-016-0292-y.

Stuttgart. 2016. Energiekonzept „Urbanisierung der Energiewende“ (Fassung vom 28.01.2016). https:// www.stuttgart.de/img/mdb/item/555993/135849.pdf. Zugegriffen: 10. Juni 2018.

UN Habitat. 2011. Cities and climate change. Global report on human settlements. London, Washington D.C.: Earthscan.

Zahran, Sammy, Himanshu Grover, Samuel D. Brody, und Arnold Vedlitz. 2008. Risk, stress, and capacity. Explaining metropolitan commitment to climate protection. Urban Affairs Review 43(4):447-474. https://doi.org/10.1177/1078087407304688. 
Zohlnhöfer, Reimut. 2008. Stand und Perspektiven der vergleichenden Staatstätigkeitsforschung. In Die Zukunft der Policy-Forschung. Theorien, Methoden, Anwendungen, Hrsg. Frank Janning, Katrin Toens, 157-174. Wiesbaden: VS. 